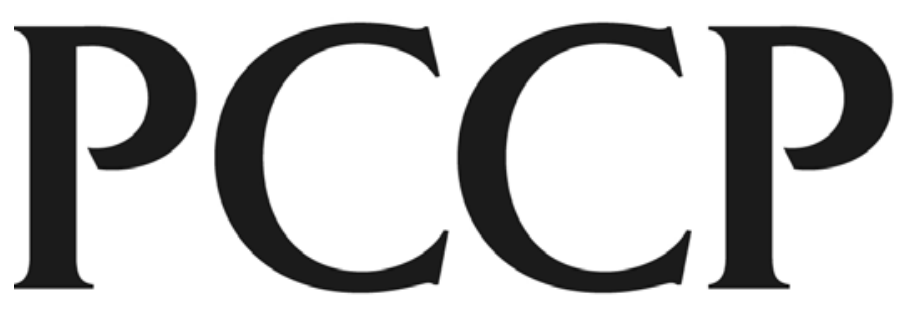

Physical Chemistry Chemical Physics

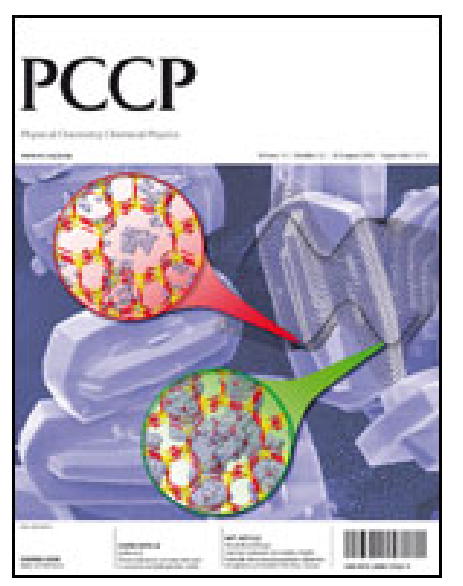

This paper is published as part of a PCCP Themed Issue on:

\author{
$\underline{\text { Water at interfaces }}$
}

Guest Editor: Martin McCoustra

Editorial

\section{Water at interfaces \\ Phys. Chem. Chem. Phys., 2008, 10, 4676 \\ DOI: $10.1039 / \mathrm{b} 812223 \mathrm{~g}$ \\ Communications}

Spectroscopic and computational evidence for $\mathrm{SO}_{2}$ ionization on $128 \mathrm{~K}$ ice surface

B. Jagoda-Cwiklik, J. P. Devlin and V. Buch, Phys. Chem.

Chem. Phys., 2008, 10, 4678

DOI: $10.1039 /$ b809839p

On "the complete basis set limit" and plane-wave methods in first-principles simulations of water

Susan B. Rempe, Thomas R. Mattsson and K. Leung, Phys.

Chem. Chem. Phys., 2008, 10, 4685

DOI: $10.1039 / \mathrm{b} 810017 \mathrm{a}$

Papers

Lattice match in density functional calculations: ice lh vs. $\beta_{\text {-Agl }}$

Peter J. Feibelman, Phys. Chem. Chem. Phys., 2008, 10, 4688

DOI: $\underline{10.1039 / b 808482 n}$
A proton between two waters: insight from fulldimensional quantum-dynamics simulations of the $\left[\mathrm{H}_{2} \mathrm{O}-\right.$ $\underline{\left.\mathrm{H}-\mathrm{OH}_{2}\right]^{ \pm} \text {cluster }}$

Oriol Vendrell and Hans-Dieter Meyer, Phys. Chem. Chem. Phys., 2008, 10, 4692

DOI: $10.1039 / \mathrm{b} 807317 \mathrm{a}$

Molecular dynamics investigation of the intrinsic structure of water-fluid interfaces via the intrinsic sampling method Fernando Bresme, Enrique Chacón and Pedro Tarazona, Phys. Chem. Chem. Phys., 2008, 10, 4704

DOI: $\underline{10.1039 / \mathrm{b} 807437 \mathrm{~m}}$

An accurate analytic representation of the water pair potential

Wojciech Cencek, Krzysztof Szalewicz, Claude Leforestier, Rob van Harrevelt and Ad van der Avoird, Phys. Chem. Chem. Phys., 2008, 10, 4716

DOI: $10.1039 / \mathrm{b} 809435 \mathrm{~g}$

Characterization of interfacial water in MOF-5

$\left(\mathrm{Zn}_{4}(\mathrm{O})(\mathrm{BDC})_{3}\right)$-a combined spectroscopic and theoretical study

K. Schröck, F. Schröder, M. Heyden, R. A. Fischer and M. Havenith, Phys. Chem. Chem. Phys., 2008, 10, 4732 DOI: $10.1039 / \mathrm{b} 807458 \mathrm{p}$

Water confined in reverse micelles-probe tool in biomedical informatics

Florin Despa, Phys. Chem. Chem. Phys., 2008, 10, 4740

DOI: 10.1039/b805699b 


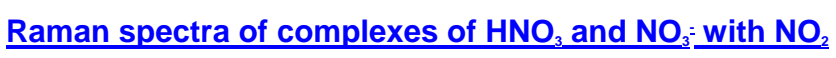
at surfaces and with $\mathrm{N}_{2} \mathrm{O}_{4}$ in solution

Michael A. Kamboures, Wytze van der Veer, R. Benny Gerber and Leon F. Phillips, Phys. Chem. Chem. Phys., 2008, 10, 4748

DOI: $10.1039 / \mathrm{b} 810081 \mathrm{k}$

Molecular level structure of the liquid/liquid interface. Molecular dynamics simulation and ITIM analysis of the water- $\mathrm{CCl}_{4}$ system

Lívia B. Pártay, George Horvai and Pál Jedlovszky, Phys. Chem. Chem. Phys., 2008, 10, 4754

DOI: $10.1039 / \mathrm{b} 807299 \mathrm{j}$

Solvent structures of mixed water/acetonitrile mixtures at chromatographic interfaces from computer simulations

Jörg Braun, Antony Fouqueau, Raymond J. Bemish and Markus Meuwly, Phys. Chem. Chem. Phys., 2008, 10, 4765 DOI: $10.1039 / \mathrm{b} 807492 \mathrm{e}$

lon spatial distributions at the liquid-vapor interface of aqueous potassium fluoride solutions

Matthew A. Brown, Raffaella D'Auria, I.-F. William Kuo, Maria J. Krisch, David E. Starr, Hendrik Bluhm, Douglas J. Tobias and John C. Hemminger, Phys. Chem. Chem. Phys., 2008, 10, 4778

DOI: $\underline{10.1039 / \mathrm{b} 807041 \mathrm{e}}$

Trapping proton transfer intermediates in the disordered hydrogen-bonded network of cryogenic hydrofluoric acid solutions

Patrick Ayotte, Sylvain Plessis and Patrick Marchand, Phys. Chem. Chem. Phys., 2008, 10, 4785

DOI: $10.1039 / \mathrm{b} 806654 \mathrm{j}$

Aqueous divalent metal-nitrate interactions: hydration versus ion pairing

Man Xu, James P. Larentzos, Mazen Roshdy, Louise J.

Criscenti and Heather C. Allen, Phys. Chem. Chem. Phys., 2008, 10, 4793

DOI: $10.1039 / \mathrm{b} 807090 \mathrm{n}$

Structure and dynamics of water at a clay surface from molecular dynamics simulation

Virginie Marry, Benjamin Rotenberg and Pierre Turq, Phys. Chem. Chem. Phys., 2008, 10, 4802

DOI: $\underline{10.1039 / \mathrm{b} 807288 d}$

\section{Proton mobility in thin ice films: a revisit}

Eui-Seong Moon, Chang-Woo Lee and Heon Kang, Phys. Chem. Chem. Phys., 2008, 10, 4814

DOI: $10.1039 / \mathrm{b} 807730 \mathrm{~b}$
Thermodynamics of water intrusion in nanoporous hydrophobic solids

Fabien Cailliez, Mickael Trzpit, Michel Soulard, Isabelle Demachy, Anne Boutin, Joël Patarin and Alain H. Fuchs, Phys. Chem. Chem. Phys., 2008, 10, 4817

DOI: $10.1039 / \mathrm{b} 807471 \mathrm{~b}$

Gas phase hydration of organic ions

Paul O. Momoh and M. Samy El-Shall, Phys. Chem. Chem. Phys., 2008, 10, 4827

DOI: $10.1039 / \mathrm{b} 809440$ n

Water photodissociation in free ice nanoparticles at 243 nm and $193 \mathrm{~nm}$

Viktoriya Poterya, Michal Fárník, Milan Ončák and Petr Slavíč ek, Phys. Chem. Chem. Phys., 2008, 10, 4835

DOI: $10.1039 / \mathrm{b} 806865 \mathrm{~h}$

Electroacoustic and ultrasonic attenuation measurements of droplet size and $\zeta$-potential of alkane-in-water emulsions: effects of oil solubility and composition Alex M. Djerdjev and James K. Beattie, Phys. Chem. Chem. Phys., 2008, 10, 4843

DOI: $10.1039 / \mathrm{b} 807623 \mathrm{e}$

Gas hydrate nucleation and cage formation at a water/methane interface

Robert W. Hawtin, David Quigley and P. Mark Rodger, Phys. Chem. Chem. Phys., 2008, 10, 4853

DOI: $10.1039 / \mathrm{b} 807455 \mathrm{k}$

Hydration water rotational motion as a source of configurational entropy driving protein dynamics. Crossovers at 150 and $220 \mathrm{~K}$

J.-M. Zanotti, G. Gibrat and M.-C. Bellissent-Funel, Phys.

Chem. Chem. Phys., 2008, 10, 4865

DOI: $10.1039 / \mathrm{b} 808217 \mathrm{k}$

Influence of wettability and surface charge on the interaction between an aqueous electrolyte solution and a solid surface

Svetlana Guriyanova and Elmar Bonaccurso, Phys. Chem. Chem. Phys., 2008, 10, 4871

DOI: $\underline{10.1039 / \mathrm{b} 806236 \mathrm{f}}$

Molecular dynamics study of hydrated imogolite 2. Structure and dynamics of confined water

Benoît Creton, Daniel Bougeard, Konstantin S. Smirnov, Jean Guilment and Olivier Poncelet, Phys. Chem. Chem. Phys., 2008, 10, 4879

DOI: $\underline{10.1039 / \mathrm{b} 803479 f}$

Assessing the performance of implicit solvation models at a nucleic acid surface

Feng Dong, Jason A. Wagoner and Nathan A. Baker, Phys.

Chem. Chem. Phys., 2008, 10, 4889

DOI: $10.1039 / \mathrm{b} 807384 \mathrm{~h}$ 
Aqueous peptides as experimental models for hydration water dynamics near protein surfaces

Cecile Malardier-Jugroot, Margaret E. Johnson, Rajesh K. Murarka and Teresa Head-Gordon, Phys. Chem. Chem.

Phys., 2008, 10, 4903

DOI: $\underline{10.1039 / \mathrm{b} 806995 \mathrm{f}}$

Melting behavior of water in cylindrical pores: carbon nanotubes and silica glasses

M. Sliwinska-Bartkowiak, M. Jazdzewska, L. L. Huang and K. E. Gubbins, Phys. Chem. Chem. Phys., 2008, 10, 4909 DOI: $\underline{10.1039 / \mathrm{b} 808246 \mathrm{~d}}$

Increased interfacial thickness of the $\mathrm{NaF}, \mathrm{NaCl}$ and $\mathrm{NaBr}$ salt aqueous solutions probed with non-resonant surface second harmonic generation (SHG)

Hong-tao Bian, Ran-ran Feng, Yan-yan Xu, Yuan Guo and Hong-fei Wang, Phys. Chem. Chem. Phys., 2008, 10, 4920 DOI: $10.1039 / \mathrm{b} 806362 \mathrm{a}$

Determination of the electron"s solvation site on $\underline{D}_{2} \mathrm{O} / \mathrm{Cu}(111)$ using $\mathrm{Xe}$ overlayers and femtosecond photoelectron spectroscopy

Michael Meyer, Julia Stähler, Daniela O. Kusmierek, Martin Wolf and Uwe Bovensiepen, Phys. Chem. Chem. Phys., 2008, 10, 4932

DOI: $10.1039 / \mathrm{b} 807314 \mathrm{~g}$

Breakdown of hydration repulsion between charged surfaces in aqueous $\mathbf{C s}^{ \pm}$solutions

Ronit Goldberg, Liraz Chai, Susan Perkin, Nir Kampf and Jacob Klein, Phys. Chem. Chem. Phys., 2008, 10, 4939

DOI: $10.1039 / \mathrm{b} 807459 \mathrm{n}$

A macroscopic water structure based model for describing charging phenomena at inert hydrophobic surfaces in aqueous electrolyte solutions Johannes Lützenkirchen, Tajana Preočanin and Nikola Kallay, Phys. Chem. Chem. Phys., 2008, 10, 4946

DOI: $\underline{10.1039 / b 807395 c}$

\section{Thermally induced mixing of water dominated} interstellar ices

Daren J. Burke, Angela J. Wolff, John L. Edridge and Wendy A. Brown, Phys. Chem. Chem. Phys., 2008, 10, 4956

DOI: $\underline{10.1039 / \mathrm{b} 807220 \mathrm{e}}$

Water hydrogen bond analysis on hydrophilic and hydrophobic biomolecule sites

Daniela Russo, Jacques Ollivier and José Teixeira, Phys.

Chem. Chem. Phys., 2008, 10, 4968

DOI: $10.1039 / \mathrm{b} 807551 \mathrm{~b}$
Hydronium and hydroxide at the interface between water and hydrophobic media

Robert Vácha, Dominik Horinek, Max L. Berkowitz and Pavel Jungwirth, Phys. Chem. Chem. Phys., 2008, 10, 4975

DOI: $\underline{10.1039 / \mathrm{b} 806432 \mathrm{f}}$

Average molecular orientations in the adsorbed water layers on silicon oxide in ambient conditions

Anna L. Barnette, David B. Asay and Seong H. Kim, Phys.

Chem. Chem. Phys., 2008, 10, 4981

DOI: $10.1039 / \mathrm{b} 810309 \mathrm{~g}$

Interfacial water structure at polymer gel/quartz interfaces investigated by sum frequency generation spectroscopy Hidenori Noguchi, Minowa Hiroshi, Taiki Tominaga, Jian Ping Gong, Yoshihito Osada and Kohei Uosaki, Phys. Chem.

Chem. Phys., 2008, 10, 4987

DOI: $10.1039 / \mathrm{b} 807297 \mathrm{n}$

\section{Co-adsorption of water and hydrogen on $\mathrm{Ni}(111)$}

Junjun Shan, Jacques F. M. Aarts, Aart W. Kleyn and Ludo B. F. Juurlink, Phys. Chem. Chem. Phys., 2008, 10, 4994 DOI: $10.1039 / \mathrm{b} 808219 \mathrm{~g}$

Water-methanol mixtures: topology of hydrogen bonded network

Imre Bakó, Tünde Megyes, Szabolcs Bálint, Tamás Grósz and Viorel Chihaia, Phys. Chem. Chem. Phys., 2008, 10, 5004 DOI: $10.1039 / \mathrm{b} 808326 f$ 


\title{
An accurate analytic representation of the water pair potential $\dagger$
}

\author{
Wojciech Cencek, ${ }^{a}$ Krzysztof Szalewicz, ${ }^{* a}$ Claude Leforestier ${ }^{b}{ }^{\text {Rob van Harrevelt }}{ }^{c}$ \\ and Ad van der Avoird ${ }^{* c}$
}

Received 3rd June 2008, Accepted 26th June 2008

First published as an Advance Article on the web 10th July 2008

DOI: $10.1039 / \mathbf{b 8 0 9 4 3 5 g}$

The $a b$ initio water dimer interaction energies obtained from coupled cluster calculations and used in the CC-pol water pair potential (Bukowski et al., Science, 2007, 315, 1249) have been refitted to a site-site form containing eight symmetry-independent sites in each monomer and denoted as CC-pol-8s. Initially, the site-site functions were assumed in a B-spline form, which allowed a precise optimization of the positions of the sites. Next, these functions were assumed in the standard exponential plus inverse powers form. The root mean square error of the CC-pol-8s fit with respect to the 2510 ab initio points is $0.10 \mathrm{kcal} \mathrm{mol}^{-1}$, compared to $0.42 \mathrm{kcal} \mathrm{mol}^{-1}$ of the CC-pol fit $\left(0.010 \mathrm{kcal} \mathrm{mol}^{-1}\right.$ compared to $0.089 \mathrm{kcal} \mathrm{mol}^{-1}$ for points with negative interaction energies). The energies of the stationary points in the CC-pol-8s potential are considerably more accurate than in the case of CC-pol. The water dimer vibration-rotation-tunneling spectrum predicted by the CC-pol-8s potential agrees substantially and systematically better with experiment than the already very accurate spectrum predicted by CC-pol, while specific features that could not be accurately predicted previously now agree very well with experiment. This shows that the uncertainties of the fit were the largest source of error in the previous predictions and that the present potential sets a new standard of accuracy in investigations of the water dimer.

\section{Introduction}

Accurate prediction of the properties of water has been one of the main goals of computational chemical physics. This field has recently been extensively discussed in ref. 1. More than a hundred interaction potentials have been proposed for water. The CC-pol potential developed in ref. 1 and 2 was the first $a b$ initio potential that accomplished the goal of the description of the complete range of water properties - from spectra of the water dimer to structure and dynamics of liquid water - at an accuracy comparable to that achieved in experiments. ${ }^{1-3}$ The CC-pol potential was a consecutive step in the development of $a b$ initio potentials, using initially symmetry-adapted perturbation theory (SAPT) ${ }^{4}$ and leading to the potentials: SAPTpp, ${ }^{5}$ SAPT-5s, ${ }^{6-8}$ and SDFT-5s. ${ }^{9}$ The CC-pol potential was based on supermolecular calculations using second-order perturbation theory with Møller-Plesset decomposition of the Hamiltonian (MP2) and the coupled-cluster method with single, double, and noniterative triple excitations [CCSD(T)]. The improvement achieved by CC-pol was, however, not due

\footnotetext{
${ }^{a}$ Department of Physics and Astronomy, University of Delaware, Newark, DE 19716,USA.E-mail: szalewic@udel.edu

${ }^{b}$ Institut Charles Gerhardt Montpellier, UMR 5253-UM2-CNRSENSCM-UM1, CTMM, Bât. 15-CC 1501, Université Montpellier 2, 34095 Montpellier Cedex 05, France

${ }^{c}$ Theoretical Chemistry, Institute for Molecules and Materials, Radboud University Nijmegen, Heijendaalseweg 135, 6525 AJ Nijmegen, The Netherlands. E-mail: A.vanderAvoird@theochem.ru.nl $\dagger$ Electronic supplementary information (ESI) available: The geometries of the stationary points predicted by the CC-pol-8s potential; Fortran source codes of the CC-pol-8s potential, 3-body nonadditive energies, and N-body induction contribution routines. See DOI: $10.1039 / \mathrm{b} 809435 \mathrm{~g}$
}

to the use of the supermolecular approach but mainly to the application of large basis sets and of extrapolations to the complete basis set (CBS) limit. Another element determining the accuracy of predictions is the ability of the functional form used to fit the potential to reproduce $a b$ initio interaction energies. Due to the computer time restrictions in molecular simulations, the analytic potentials cannot have too complicated forms. In fact, the SAPT-pp potential-which was an expansion into products of angular Wigner functions and of exponential and inverse power functions of the center-of-mass distance - was too complicated for molecular simulations. The other potentials listed above utilized a sum of isotropic site-site functions with the set of five symmetry-distinct sites developed in ref. 6 . The SDFT $-5 s^{9}$ and CC-pol ${ }^{1,2}$ potentials had also an explicit polarizable center in order to streamline applications in molecular simulations; this extension had only a very small influence on the quality of the fit. It became clear after the development of the CC-pol potential that a point has been reached where the limited accuracy of the fitting function became a more restrictive factor than the uncertainties of $a b$ initio points. This is clearly shown by the fact that the root mean square error (RMSE) of the CC-pol fit relative to the $a b$ initio interaction energies is $0.09 \mathrm{kcal} \mathrm{mol}^{-1}$ for negative energies, whereas the estimated uncertainty of the interaction energies is $0.05 \mathrm{kcal} \mathrm{mol}^{-1}$.

The aim of the present paper is to improve the flexibility of the functional form up to the point where the uncertainty originating from this source will be significantly smaller than the uncertainties of $a b$ initio points. This goal could certainly have been achieved with the form utilized in SAPT-pp, but as mentioned above, this would make molecular simulations 
difficult. One may view SAPT-pp as a site-site potential with only one site (the monomer's center of mass) per molecule but with extremely anisotropic site-site functions. Thus, another option would be to use a small number of sites per molecule and make the site-site functions anisotropic, but only using very simple anisotropic terms. Still, for such a potential it would be difficult to program analytic derivatives needed in molecular dynamics simulations. Therefore, we have decided to use the same general form as in the SDFT-5s and CC-pol potentials but increase the flexibility of the site-site functions and/or the number of sites. Another option could be to use in addition to a sum of site-site functions also functions depending on more than one site-site coordinate. Such a form has been successfully used in the potentials developed by Huang et al. ${ }^{10,11}$ However, it is not clear how to use such potentials in molecular simulations and how to assure the correct longrange asymptotic behavior.

\section{Generation of the fit}

\section{A Exponential plus inverse power form of the fit}

The functional form of the final fit developed in this work is identical with that of the CC-pol fit, ${ }^{1,2}$ namely

$$
V=\sum_{a \in A} \sum_{b \in B} u_{a b}\left(r_{a b}\right)+V_{2}^{\text {ind }}(A, B)
$$

where $u_{a b}$ are site-site potentials depending only on distances $r_{a b}$ between sites associated with monomers $A$ and $B$ and $V_{2}^{\text {ind }}(A, B)$ represents the induction interaction. The functions $u_{a b}$ have the following general form

$$
\begin{aligned}
u_{a b}\left(r_{a b}\right)= & \exp \left(-\beta_{a b} r_{a b}\right) \sum_{m=0}^{3} c_{m}^{a b} r_{a b}^{m}+f_{1}\left(\delta_{1}^{a b}, r_{a b}\right) \frac{q_{a} q_{b}}{r_{a b}} \\
& +\sum_{n=6,8,10} f_{n}\left(\delta_{n}^{a b}, r_{a b}\right) \frac{C_{n}^{a b}}{r_{a b}^{n}},
\end{aligned}
$$

i.e., each function may contain an exponential part times a polynomial, an electrostatic term with site charges $q_{a}$ and $q_{b}$, and $r_{a b}^{-n}$ terms reproducing the asymptotic dispersion interaction and a part of the asymptotic induction interaction that is not accounted for by $V_{2}^{\text {ind }}(A, B)$. The damping functions $f_{n}(\delta, r)$ of the Tang-Toennies form,

$$
f_{n}(\delta, r)=1-e^{-\delta r} \sum_{m=0}^{n} \frac{(\delta r)^{m}}{m !},
$$

partly recover the charge-overlap effects. All the long-range asymptotic terms of the CC-pol fit were unchanged in the present work. Although one could have hoped that simple extensions of the functional form specified above-like increasing the polynomial order in the exponential term or using multiple exponentials - will lead to much more accurate fits, all our attempts in this direction resulted only in minor improvements of accuracy. As we will show below, this accuracy can be more significantly improved by using a different type of site-site functions, but such an improvement comes at the price of excessive undulations between the grid points. Thus, the form defined above turned out to be exceptionally robust. Note that although this paper discusses only the properties of the water dimer, the potential form of eqn (1) can be used in simulations of larger clusters and condensed phases if the term $V_{2}$ is replaced by the analogous $N$-body polarization term, see ref. 1 and 3 .

To generate all the fits discussed in this paper, we used the set of 2510 water dimer geometries from ref. 6, the same geometries as used to construct the CC-pol potential in ref. 1 and 2. We have also utilized the results of the $a b$ initio MP2 and $\operatorname{CCSD}(\mathrm{T})$ calculations from the latter references. However, in the process of this work we found a minor error in the extrapolations of the MP2 results in ref. 1 and 2. We have therefore repeated the extrapolations according to eqn (3) in ref. 1 . The differences relative to ref. 1 and 2 were very small, generally on the order of $0.01 \mathrm{kcal} \mathrm{mol}^{-1}$. In the most important region of -5 to $+5 \mathrm{kcal} \mathrm{mol}^{-1}$, the average absolute deviation from the correct values amounts to only $0.004 \mathrm{kcal} \mathrm{mol}^{-1}$, with a standard deviation of $0.004 \mathrm{kcal}$ $\mathrm{mol}^{-1}$. This can be compared with an estimated absolute accuracy of the ab initio energies, equal to about $0.05 \mathrm{kcal}$ $\mathrm{mol}^{-1}$, and with the accuracy of the CC-pol fit itself, which was $0.09 \mathrm{kcal} \mathrm{mol}^{-1}$ in the region of -5 to $0 \mathrm{kcal} \mathrm{mol}^{-1}$. Although these deviations were clearly inconsequential at the accuracy level of ref. 1 and 2, such deviations could become relevant at the levels reached in the present work.

\section{B Spline form of the fit}

In order to find out how various choices of sites and forms of the functions $u_{a b}\left(r_{a b}\right)$ influence the accuracy of the fit, we have decided to replace the expansion of eqn (2) by spline expansions. This choice enables to achieve completeness of the 'basis set' for each $u_{a b}\left(r_{a b}\right)$ and also, as discussed in more detail below, leads to more effective optimizations of the site positions.

The B-splines of order $k$ of one variable $r$ are defined recursively as

$$
B_{i, 1}(r)=\left\{\begin{array}{lc}
1, & t_{i} \leq r<t_{i+1} \\
0, & \text { otherwise }
\end{array}\right.
$$

and

$$
B_{i, k}(r)=\frac{r-t_{i}}{t_{i+k-1}-t_{i}} B_{i, k-1}(r)+\frac{t_{i+k}-r}{t_{i+k}-t_{i+1}} B_{i+1, k-1}(r),
$$

where $\left\{t_{i}\right\}, i=1,2, \ldots, N$, is a nondecreasing sequence of values (knots) of the argument $r . B_{i, k}$ is a piecewise polynomial of degree $k-1$ having nonzero values only in the interval $t_{i} \leq$ $r<t_{i+k}$. Following Johnson et al., ${ }^{12}$ we distributed the first $k$ knots at $r^{\min }$, the last $k$ knots at $r^{\max }$, and the remaining $N-2 k$ knots uniformly on a logarithmic scale between $r^{\text {min }}$ and $r^{\max }$. For such a knot distribution, the nonvanishing splines $B_{i, k}$ are those with $i=1,2, \ldots, N_{\mathrm{spl}}$, where $N_{\mathrm{spl}}=N$ $-k$. The role of the multiple knots at $r^{\min }$ and $r^{\max }$ is to ensure that each of the $N-2 k+1$ segments, into which the whole range of $r$ is divided, supports the same number $(k)$ of nonvanishing splines and so the expected accuracy is uniform in the whole range. The use of the logarithmic distribution is arbitrary and not as well motivated here as in the atomic calculations of ref. 12. What is relevant, however, is the fact that the distribution scheme remains the same for any number and location of the sites, which makes their optimization 
unbiased. The values of $r^{\min }$ and $r^{\max }$, different for each site-site pair and denoted from now on as $r_{a b}^{\min }$ and $r_{a b}^{\max }$, were chosen as $0.01 a_{0}$ smaller and $0.01 a_{0}$ larger, respectively, than the smallest and largest distance between this particular pair of sites occurring in the set of 2510 fitted points. The functional form of the B-spline fit is that of eqn (1), with $u_{a b}$ expanded as

$$
u_{a b}\left(r_{a b}\right)=\sum_{i=1}^{N_{\mathrm{spl}}} b_{i}^{a b} B_{i, k}^{a b}\left(r_{a b}\right),
$$

where the linear coefficients $b_{i}^{a b}$ are found by the least-square fitting of $V$ in eqn (1) to the $a b$ initio interaction energies $E_{\text {int }}$ at the 2510 grid points, using the same weighting factors as in the CC-pol fit, i.e., $w=1 /\left(E_{\text {int }}+5.5 \mathrm{kcal} \mathrm{mol}^{-1}\right)^{2}$ (the shift is related to the well depth of $5.0 \mathrm{kcal} \mathrm{mol}^{-1}$ ). Since the B-splines form a complete basis set, the accuracy of this fit is restricted only by the number and positions of the sites and by the number of the splines. Of course, without adding proper shortrange and asymptotic terms, the fit is undefined for any water dimer configuration where a particular site-site distance does not belong to the interval $\left(r_{a b}^{\min }, r_{a b}^{\max }\right)$, but this fact is not relevant for our present purposes.

It should be noted that, when the value of $N_{\text {spl }}$ is sufficiently large, for some variables $r_{a b}$ in eqn (6) there will exist $k$ (or more) consecutive segments (created by dividing the whole range from $r_{a b}^{\min }$ to $r_{a b}^{\max }$ with the knots) not covered by any of the 2510 data points. Since each spline of the $k$ th order spans $k$ consecutive segments, the least-square problem then becomes singular, because the particular function (or functions) $B_{i, k}^{a b}$ which differs from zero only on these segments vanishes at all data points. To avoid this problem, our fitting program first analyzes the standard distribution of the spline knots in relation to the data points for each variable and, if needed, reduces the number of knots $N$ until only $k-1$ consecutive segments without any data exist (this is due to the fact that with smaller $N$ the segments become larger). Since the reduction of $N$ lowers $N_{\text {spl }}$ for this particular variable $r_{a b}$, for the largest of our expansions the total number of splines (the order of the least-square problem matrix) is also slightly reduced.

\section{Convergence in the number of splines}

First, we tested the convergence of the B-spline fits with the number of splines used for each site-site distance. The tests were performed for the numbers of sites varying from 2 to 5 and all the site positions were taken from ref. 6 (the same positions as used in the CC-pol fit). Results of these tests are presented in Table 1 for $k=3$. We also tried splines of higher orders but the dependence of the results on $k$ is not very significant for the purpose of this work. The values of root mean square errors (RMSE) corresponding to the very large numbers of splines are particularly interesting because such values provide estimates of the limits achievable with a given site configuration using the best possible functional form of the fit. In particular, we can see that the RMSE values for all 2510 points and for points with negative interaction energies, amounting to 0.42 and $0.089 \mathrm{kcal} \mathrm{mol}^{-1}$, respectively, achieved by the original 5-site CC-pol fit [1] could have been significantly improved by choosing more flexible site-site functions. The most accurate fit of Table 1 reduces the corresponding RMSE's to 0.17 and $0.038 \mathrm{kcal} \mathrm{mol}^{-1}$, i.e., by more than a factor of two. One can also see that an increase of $N_{\text {spl }}$ above 42 leads to relatively small decreases of RMSE's. By contrast, the decreases are substantial with each new site added. Clearly, in order to further increase the accuracy of the fit, one needs more than five sites.

Moreover, we have found that although adding more splines allows one to reproduce the energies of the fitted data set better and better, the fit quality between the grid points worsens starting from some value of $N_{\text {spl. }}$. This is illustrated in Table 2, where we examine how various 5-site fits reproduce the barriers in the potential, i.e., the energy differences between the global minimum and the nine other stationary points on the dimer potential energy surface. The geometries of all the points were those obtained in ref. 1 by searching the CC-pol surface. For $N_{\mathrm{spl}} \leq 42$, the spline fits reproduce the barriers with an accuracy very similar to that of the CC-pol fit, and the RMSE is almost constant between $N_{\text {spl }}=12$ and $N_{\text {spl }}=42$. For $N_{\mathrm{spl}}>42$, the quality of predictions rapidly deteriorates. Evidently, the flexibility of the B-splines in reproducing the fitted energies is associated with an oscillatory behavior between the fitted data points, while the CC-pol fit apparently behaves more smoothly. Therefore, we concluded that the CCpol functional form is preferred over the B-spline form and the best way to improve the accuracy of the fit is to use more sites. We also found, however, that for reasons described below, the B-spline fits are useful for optimization of the site positions.

\section{Optimization of site positions}

The original CC-pol $\mathrm{fit}^{1,2}$ depends on five symmetry-unique sites. The first two sites are fixed at the positions of the $\mathrm{O}$ and $\mathrm{H}$ atoms. The position of the third site (denoted by D1) was

Table 1 Convergence of the $k=3 \mathrm{~B}$-spline site-site fits for the water dimer with the number of symmetry-unique sites $N_{\mathrm{S}}$ (the total number of sites is given in parentheses) and with the number of splines for each site-site distance, $N_{\text {spl. }}$. For each value of $N_{\mathrm{S}}$ the numbers in the first row are the total RMSE, the numbers in the second row are the RMSE for points with negative interaction energies (both in kcal mol ${ }^{-1}$ ). The corresponding errors of the CC-pol fit are 0.42 and $0.089 \mathrm{kcal} \mathrm{mol}^{-1}$. The site positions are the same as in the CC-pol fit ${ }^{1,2}$

\begin{tabular}{|c|c|c|c|c|c|}
\hline$N_{\mathrm{S}} \backslash N_{\mathrm{spl}}$ & 12 & 22 & 42 & 82 & 122 \\
\hline \multirow[t]{2}{*}{ 2(3) } & 1.901 & 1.857 & 1.831 & 1.773 & 1.746 \\
\hline & 0.564 & 0.557 & 0.530 & 0.501 & 0.482 \\
\hline \multirow[t]{2}{*}{$3(5)$} & 0.599 & 0.591 & 0.570 & 0.536 & 0.505 \\
\hline & 0.193 & 0.170 & 0.162 & 0.151 & 0.142 \\
\hline \multirow[t]{2}{*}{$4(6)$} & 0.508 & 0.482 & 0.434 & 0.386 & 0.318 \\
\hline & 0.145 & 0.128 & 0.115 & 0.102 & 0.092 \\
\hline \multirow[t]{2}{*}{$5(8)$} & 0.347 & 0.327 & 0.290 & 0.225 & 0.166 \\
\hline & 0.094 & 0.074 & 0.059 & 0.047 & 0.038 \\
\hline
\end{tabular}


Table 2 Stationary-point energies relative to the potential minimum (barriers) at the geometries found on the CC-pol potential surface ${ }^{1,2}$ (identical geometries are used for all fits and for $a b$ initio calculations). All the fits are based on the same five sites from ref. 6 . The barriers predicted by the CC-pol fit and by B-spline fits with $N_{\text {spl }}$ splines for each site-site distance are compared with the calculated ab initio values (in $\mathrm{cm}^{-1}$ ). See ref. 13 for a description and numbering of the stationary points

\begin{tabular}{|c|c|c|c|c|c|c|c|}
\hline No. & CC-pol & $N_{\mathrm{spl}}=12$ & $N_{\mathrm{spl}}=22$ & $N_{\mathrm{spl}}=42$ & $N_{\mathrm{spl}}=82$ & $N_{\mathrm{spl}}=122$ & $A b$ initio \\
\hline 2 & 187.9 & 200.1 & 191.2 & 193.4 & 142.6 & 151.1 & 198.9 \\
\hline 3 & 189.3 & 222.3 & 210.3 & 208.8 & 160.9 & 160.4 & 213.8 \\
\hline 4 & 211.1 & 211.7 & 198.0 & 173.0 & 130.2 & 124.1 & 256.7 \\
\hline 5 & 330.5 & 333.0 & 276.3 & 282.8 & 220.3 & 160.6 & 344.1 \\
\hline 6 & 351.6 & 401.5 & 326.3 & 359.9 & 314.2 & 162.4 & 365.2 \\
\hline 7 & 746.4 & 642.7 & 683.6 & 689.2 & 637.0 & 659.2 & 702.2 \\
\hline 8 & 1333.9 & 1293.5 & 1288.5 & 1288.0 & 1424.7 & 1590.6 & 1297.1 \\
\hline 9 & 731.2 & 691.6 & 674.1 & 690.2 & 618.3 & 591.5 & 702.6 \\
\hline 10 & 1005.3 & 1078.9 & 1029.3 & 1021.7 & 983.1 & 1003.0 & 1023.2 \\
\hline $\mathrm{RMSE}^{b}$ & 29.1 & 33.9 & 34.8 & 35.4 & 87.7 & 148.4 & \\
\hline
\end{tabular}

thoroughly optimized in ref. 6. However, the positions of the remaining two sites (D2 and D3) were only crudely optimized in ref. 6. The aim of the present work was to obtain well optimized positions of these two sites as well as of additional sites added until a satisfactory fit quality was achieved.

The optimization of the site positions using the original functional form of eqns (1)-(3) is difficult because the nonlinear least-square fitting process is time consuming and prone to end in one of the numerous local minima. Therefore, for the purpose of the site optimization, we decided to use B-splines to represent the functional dependence on each site-site distance. If the distribution of the spline knots is fixed, the minimization of the RMSE of such a fit represents a linear least-square problem and leads to an unambiguous solution at each site configuration. Of course, the search for the optimal site positions is still nonlinear.

In the optimizations of site positions, we used 42 splines for each site-site pair. As shown above, this expansion length does not lead to an excessive oscillatory behavior while providing a high fit accuracy and being relatively fast. We started from the 3 sites of the original CC-pol fit (O, H, D1, kept fixed) and added the next site, optimizing its position via a minimization of the RMSE of the fit defined by eqns (1) and (6) on the standard set of 2510 water dimer geometries. Then, the next site was added and optimized while keeping the positions of the other sites frozen. The actual optimization was carried out by checking every point of a 3-D grid of $X, Y$, and $Z$ coordinates, where $X$ and $Y$ each spanned 16 values between 0.0 and $2.0 a_{0}$ and $Z$ spanned 23 values between -1.5 and 1.0 $a_{0}$ (since $Y Z$ and $X Z$ are symmetry planes of the molecule, only non-negative values of $X$ and $Y$ need to be tested). Then, a smaller sub-grid was built around the crude position of the minimum just found and the process was continued until each coordinate was converged to at least two significant digits. Table 3 contains the optimized positions of the $4 \mathrm{th}, 5 \mathrm{th}, 6 \mathrm{th}$, 7 th, and 8 th site (with the first 3 sites included for completeness), along with the RMSE values of the corresponding B-spline fits.

\section{E Final fitting}

Our final recommended fit was obtained using 8 symmetryunique sites ( 25 sites in total) from Table 3 and the same functional form as the original CC-pol fit, i.e., eqns (1)-(3). As before, the electrostatic term was used only to describe the interaction of the $\mathrm{O}, \mathrm{H}$, and $\mathrm{D} 1$ sites, while the induction-dispersion term was restricted to the $\mathrm{O}$ and $\mathrm{H}$ sites. The exponential term was used in all the sites, including the D1 site, where it was omitted in the CC-pol fit. Our new fit has, therefore, the following parameters: 18 site coordinates, 3 site charges (located on $\mathrm{O}, \mathrm{H}$, and D1), $9 C_{n}^{a b}$ constants, 15 damping parameters $\left(6 \delta_{1}, 3 \delta_{6}, 3 \delta_{8}, 3 \delta_{10}\right), 36$ values of $\beta_{a b}$, and 144 linear parameters $c_{m}^{a b}$. The asymptotic part of the fit was taken from the CC-pol fit, i.e., the site charges and $C_{n}^{a b}$ constants were not changed. Hence, the actual fitting procedure was restricted to 51 nonlinear and 144 linear parameters. We performed the process several dozen times, starting from different initial values of the parameters, and tested the resulting fits for the values of RMSE and for the asymptotic and short-distance behavior. In particular, the latter varied dramatically and eliminated most of the fits from the consideration (see later in this section).

The final fit, denoted as "exp $+1 / r$ " in the last row of Table 3, has the RMSE of $0.1037 \mathrm{kcal} \mathrm{mol}^{-1}$ for all the 2510 dimer geometries and $0.0103 \mathrm{kcal} \mathrm{mol}^{-1}$ for those with $E_{\mathrm{int}}<$ 0 . Table 4 compares the performance of various fits containing the optimized sites with the original CC-pol fit on the set of 9 stationary points. Similarly as for a large number of splines $N_{\text {spl }}$ (see Table 2), increasing the number of sites beyond a certain value $\left(N_{\mathrm{S}}=6\right)$ leads to the progressive deterioration of the stationary point description by the spline fits. On the other hand, the RMSE values for the exponential fits decrease smoothly which confirms that these fits perform relatively better than the spline fits in the areas between the fitted points.

Fits of the form of eqns (1)-(3) typically exhibit unphysical behavior at small distances by collapsing to $-\infty$, which causes problems in applications like molecular simulations. This collapsing is inconsequential, however, if it starts at distances where the repulsive barrier is high enough to prevent the simulation from choosing the path towards still smaller distances. Let $V 0(R)$ denote the minimum (with respect to all the angles) interaction energy at a given distance $R$ between the centers of masses (COM) of the two water molecules. We selected, among many almost equivalent (in terms of the accuracy and asymptotic behavior) fits, the one having the highest maximum value (barrier height) on the $V 0(R)$ plot. 
Table 3 Optimized positions (in $a_{0}$ ) of sites 4-8 (the first three sites also given for completeness) and the values of RMSE (in kcal mol ${ }^{-1}$ ) for the spline (spl) or exponential plus inverse powers of $r_{a b}(\exp +1 / r)$ fits containing the given number of symmetry-unique sites, $N_{\mathrm{S}}$. The number in parentheses after $N_{\mathrm{S}}$ denotes the total number of sites. All spline fits are with $N_{\text {spl }}=42$

\begin{tabular}{|c|c|c|c|c|c|c|c|}
\hline Site & $N_{\mathrm{S}}$ & $X$ & $Y$ & $Z$ & Fit & RMSE & $\operatorname{RMSE}\left(E_{\text {int }}<0\right)$ \\
\hline $\mathrm{O}$ & $1(1)$ & 0.0 & 0.0 & 0.1255334885 & & & \\
\hline $\mathrm{H}$ & $2(3)$ & 1.4536519622817 & 0.0 & -0.9961538357 & $\mathrm{spl}$ & 1.8286 & 0.5289 \\
\hline D1 & $3(5)$ & 0.0 & 0.2067213 & -0.2462589474 & $\mathrm{spl}$ & 0.5697 & 0.1617 \\
\hline N1 & $4(9)$ & 0.90 & 0.053 & 0.64 & $\mathrm{spl}$ & 0.2529 & 0.0556 \\
\hline \multirow[t]{2}{*}{$\mathrm{N} 2$} & $5(13)$ & 1.29 & 0.14 & -0.91 & spl & 0.1069 & 0.0217 \\
\hline & & & & & $\exp +1 / r$ & 0.1483 & 0.0326 \\
\hline \multirow[t]{2}{*}{ N3 } & $6(17)$ & 0.56 & 0.24 & -0.48 & $\mathrm{spl}$ & 0.0754 & 0.0127 \\
\hline & & & & & $\exp +1 / r$ & 0.1307 & 0.0168 \\
\hline \multirow[t]{2}{*}{ N4 } & $7(21)$ & 0.91 & 0.21 & -0.68 & $\mathrm{spl}$ & 0.0378 & 0.0093 \\
\hline & & & & & $\exp +1 / r$ & 0.1152 & 0.0124 \\
\hline \multirow[t]{2}{*}{ N5 } & $8(25)$ & 1.48 & 0.26 & -0.62 & $\mathrm{spl}$ & 0.0259 & 0.0065 \\
\hline & & & & & $\exp +1 / r$ & 0.1037 & 0.0103 \\
\hline
\end{tabular}

This height amounts to about $59 \mathrm{kcal} \mathrm{mol}^{-1}$, while for CC-pol it was about $46 \mathrm{kcal} \mathrm{mol}^{-1}$.

\section{Stationary points}

The parameters of the global minimum in the CC-pol-8s potential are listed in Table 5 and compared to literature data. Both the depth of the minimum, $D_{\mathrm{e}}$, and the geometry are very similar to that predicted by CC-pol. This close agreement, despite the differences in RMSE discussed earlier, is due to the fact that the minimum region is very densely sampled in the set of 2510 grid points and was therefore very well fitted already by the CC-pol potential. The (small) discrepancies between $\mathrm{CC}$-pol-8s results and the benchmark calculations from ref. 13 and 14 are due almost exclusively to the use of a rigid monomer geometry in CC-pol-8s, as it will be shown below, see also ref. 18. In all cases, theory describes the water dimer minimum much more accurately than do experiments.

The CC-pol-8s fit was next used to predict geometries and energies relative to the minimum of stationary points (barriers) on the water dimer surface. Since the benchmark barriers of Tschumper et al., ${ }^{13}$ denoted by TLHVSQ, were obtained by optimizing all degrees of freedom and CC-pol-8s fit assumes rigid monomers, we had to account for the flexibility correc- tion to make meaningful comparisons at the current level of accuracy. This correction was estimated at each of the stationary points in the following way. We calculated the differences

$$
E_{\mathrm{R} \rightarrow \mathrm{F}}=E_{\mathrm{TF}}-E_{\mathrm{TR}}
$$

using the CCSD(T) level of theory and the aug-cc-pVTZ basis set with additional $3 \mathrm{~s} 2 \mathrm{p} 1 \mathrm{~d}$ bond functions ${ }^{5}$ located at the midpoint between the centers of mass (COM) of the two water molecules. In the equation above, $E_{\mathrm{TF}}$ is the total water dimer electronic energy at the geometry (optimized by Tschumper et $a l .{ }^{13}$ ) of a flexible-monomer stationary point and $E_{\mathrm{TR}}$ is the corresponding energy at the rigid-monomer geometry. The latter geometry was obtained from the TF geometry by placing the rigid $\mathrm{CC}$-pol monomers at the same COM distance as in $\mathrm{TF}$ and with overlapping lines connecting the $\mathrm{O}$ atom with the center of the $\mathrm{H}-\mathrm{H}$ segment. This choice is not unique, however. Different (reasonable) choices lead to significantly different geometries only if the monomers are strongly distorted from isosceles triangles, which is not the case at the stationary points. The quantity defined in eqn (7) represents the stabilization energy at a given dimer geometry due to the relaxation of the monomers. Subtracting, at each geometry, the value of this quantity for the minimum configuration, we obtain the relative

Table 4 Stationary-point energies relative to the potential minimum (barriers) at the geometries found on the CC-pol potential surface ${ }^{1,2}$ (identical geometries are used for all fits and for ab initio calculations). The barriers predicted by the CC-pol fit, by B-spline fits (spl), and by exponential plus inverse powers of $r_{a b}(\exp +1 / r)$ fits are compared with the calculated ab initio values (in $\mathrm{cm}^{-1}$ ). The CC-pol fit employs its original sites, all the other fits use sites optimized in the present work. All the spline fits contain 42 splines for each site-site pair. See ref. 13 for a description and numbering of the stationary points

\begin{tabular}{|c|c|c|c|c|c|c|c|c|c|c|}
\hline \multirow[b]{2}{*}{ No. } & \multicolumn{3}{|l|}{$\underline{5 s}$} & \multicolumn{2}{|l|}{$6 \mathrm{~s}$} & \multicolumn{2}{|l|}{$7 \mathrm{~s}$} & \multicolumn{2}{|l|}{$8 \mathrm{~s}$} & \multirow[b]{2}{*}{ Ab initio } \\
\hline & CC-pol & spl & $\exp +1 / r$ & spl & $\exp +1 / r$ & spl & $\exp +1 / r$ & spl & $\exp +1 / r$ & \\
\hline 2 & 187.9 & 198.4 & 199.8 & 201.2 & 196.7 & 198.9 & 197.8 & 195.8 & 198.7 & 198.9 \\
\hline 3 & 189.3 & 211.7 & 214.1 & 215.4 & 209.4 & 212.7 & 211.4 & 211.8 & 212.7 & 213.8 \\
\hline 4 & 211.1 & 266.6 & 256.0 & 259.6 & 258.3 & 255.2 & 256.6 & 257.4 & 258.4 & 256.7 \\
\hline 5 & 330.5 & 351.0 & 352.2 & 342.8 & 349.7 & 333.9 & 345.7 & 327.3 & 345.2 & 344.1 \\
\hline 6 & 351.6 & 374.3 & 363.2 & 362.5 & 365.4 & 356.2 & 365.7 & 354.2 & 368.0 & 365.2 \\
\hline 7 & 746.4 & 693.3 & 704.9 & 697.3 & 699.8 & 697.5 & 698.6 & 695.2 & 698.9 & 702.2 \\
\hline 8 & 1333.9 & 1300.5 & 1314.8 & 1294.0 & 1287.2 & 1296.3 & 1292.1 & 1292.9 & 1295.1 & 1297.1 \\
\hline 9 & 731.2 & 706.5 & 703.7 & 707.5 & 695.4 & 699.0 & 700.4 & 695.6 & 700.7 & 702.6 \\
\hline 10 & 1005.3 & 1035.8 & 1022.4 & 1025.7 & 1024.2 & 1022.8 & 1026.0 & 1018.3 & 1026.6 & 1023.2 \\
\hline $\mathrm{RMSE}^{b}$ & 29.1 & 7.4 & 6.6 & 3.1 & 4.9 & 5.0 & 2.6 & 7.9 & 2.2 & \\
\hline
\end{tabular}


Table 5 Parameters of the global minimum of water dimer. Distances in $\AA$, angles in degrees, energies in $\mathrm{kcal}^{\mathrm{mol}}{ }^{-1}$. See ref. 13 for definitions and atom labeling. Square brackets around $r_{\mathrm{H}_{2} \mathrm{O}_{1}}$ indicate that this parameter was fixed during the optimization

\begin{tabular}{|c|c|c|c|c|c|}
\hline & $r_{\mathrm{O}_{1} \mathrm{O}_{2}}$ & $r_{\mathrm{H}_{2} \mathrm{O}_{1}}$ & $\theta_{\mathrm{O}_{2} \mathrm{O}_{1} \mathrm{H}_{2}}$ & $\theta_{\mathrm{O}_{1} \mathrm{O}_{2} \text { Bisec }}$ & Energy \\
\hline CC-pol-8s & 2.9105 & {$[0.9716]$} & 5.98 & 121.88 & $-5.104^{a}$ \\
\hline CC-pol & 2.9107 & {$[0.9716]$} & 6.48 & 122.66 & $-5.109^{b}$ \\
\hline $\operatorname{CCSD}(\mathrm{T})^{c}$ & 2.909 & 0.9653 & 4.47 & 124.92 & $-5.04 \pm 0.08$ \\
\hline $\operatorname{CCSD}(\mathrm{T})+\delta_{\mathrm{BD}(\mathrm{TQ})}{ }^{d}$ & & & & & $-5.02 \pm 0.08$ \\
\hline $\operatorname{CCSD}(T)^{e}$ & $2.912 \pm 0.005$ & 0.9639 & 5.5 & 124.4 & $-5.02 \pm 0.05$ \\
\hline SAPT $-5 s^{f}$ & 2.9549 & [0.9716] & 6.36 & 127.16 & -4.861 \\
\hline SAPT $-5 s t^{g}$ & 2.9244 & {$[0.9716]$} & 6.95 & 121.5 & -5.029 \\
\hline VRT(ASP-W) ${ }^{h}$ & 2.924 & {$[0.9572]$} & -2.06 & 131.5 & -4.91 \\
\hline VRT(ASP-W)II ${ }^{i}$ & 2.952 & {$[0.9572]$} & -2.40 & 131.5 & -4.85 \\
\hline Experiment & $2.91 \pm 0.005^{j}$ & & $-1.0 \pm 10^{k}$ & $123.0 \pm 10^{k}$ & $-5.00 \pm 0.7^{j}$ \\
\hline
\end{tabular}

${ }^{a}$ The interaction energy given by the fit is identical to the number of digits quoted with the energy computed ab initio. ${ }^{b}$ Energy calculated ab initio by us using eqn (3) in ref. 1 . The value $-5.103 \mathrm{kcal} \mathrm{mol}^{-1}$ listed in ref. 1 is different due to the extrapolation error discussed in the text. The fit at the minimum predicts $-5.097 \mathrm{kcal} \mathrm{mol}^{-1}$. $^{c}$ Tschumper et al., ref. 13, CP-uncorrected optimization using CCSD(T) method in TZ2P(f,d) + dif basis set. Energy computed at the CCSD(T) level with CBS-extrapolated MBPT2 component and with a correction for core effects. ${ }^{d}$ As in footnote $b$ plus a correction $\delta E_{\mathrm{BD}(\mathrm{TO})}$ for effects of higher excitations from the Brueckner method, see ref. 13. ${ }^{e}$ Klopper et al., ref. 14, 2-dimensional optimization using CBS-extrapolated CCSD(T). ${ }^{f}$ Mas et al., ref. 6, SAPT-5s fit to 2510 energies computed using SAPT method. ${ }^{g}$ Ref. 7 , SAPT-5s fit with a subset of parameters tuned to reproduce experimental VRT spectrum of the dimer. ${ }^{h}$ Ref. 15 , ASP-W potential with subset of parameters tuned to reproduce experimental VRT spectrum of the dimer. ${ }^{i}$ Ref. 16, refit of VRT(ASP-W) potential. ${ }^{j}$ Mixed experimental-theoretical estimate from ref. 6. ${ }^{k}$ Odutola and Dyke, ref. 17.

flexibility corrections $\Delta E_{\mathrm{R} \rightarrow \mathrm{F}}$, i.e., the correction to the barriers, listed in the last column of Table 6 .

The barriers given by the CC-pol-8s potential are compared in Table 7 with predictions from other potentials, in particular with the benchmark energies of TLHVSQ obtained by full, 12dimensional (12D) geometry optimizations at the CCSD(T) level and using extrapolations of augmented correlation-consistent basis sets (aug-cc-pVXZ, X $=2$ to 6). To estimate the accuracy of the CC-pol-8s fit, the ab initio energies calculated (in the same way as for the 2510 fitted points) at the CC-pol-8s geometries are also listed. It can be seen that all the discrepancies between the $a b$ initio and fitted energies are smaller than $4 \mathrm{~cm}^{-1}$ (the relative RMSE is just $2.4 \mathrm{~cm}^{-1}$ ), a dramatic improvement in comparison with the CC-pol fit quality in Table 4, where the discrepancies are larger than $40 \mathrm{~cm}^{-1}$ at points 4 and 7 and the RMSE is as large as $29 \mathrm{~cm}^{-1}$. The CCpol-8s barriers are significantly closer to the benchmark TLHVSQ values than the CC-pol barriers (RMSE of 46.6 $\mathrm{cm}^{-1}$ compared to $63.0 \mathrm{~cm}^{-1}$ ), although in this case the

Table 6 Total water dimer energies (in $E_{\mathrm{h}}$ ) at stationary points: at Tschumper et al.'s original geometries, $E_{\mathrm{TF}},{ }^{13}$ and at the same intermolecular geometries but with rigid monomers obtained as described in the text, $E_{\mathrm{TR}}$. All energies calculated at the $\operatorname{CCSD}(\mathrm{T})$ level using the aug-cc-pVTZ basis set with $3 s 2 p 1 d$ bond functions. $E_{\mathrm{R} \rightarrow \mathrm{F}}=E_{\mathrm{TF}}-$ $E_{\mathrm{TR}}$ (in $\mathrm{cm}^{-1}$ ) is the flexibility effect at a given stationary point and $\Delta E_{\mathrm{R} \rightarrow \mathrm{F}}(m)=E_{\mathrm{R} \rightarrow \mathrm{F}}(m)-E_{\mathrm{R} \rightarrow \mathrm{F}}(1)\left(\right.$ in $\left.\mathrm{cm}^{-1}\right)$ is the flexibility correction for a given barrier

\begin{tabular}{llllr}
\hline$m$ & $E_{\mathrm{TR}}$ & $E_{\mathrm{TF}}$ & $E_{\mathrm{R} \rightarrow \mathrm{F}}$ & $\Delta E_{\mathrm{R} \rightarrow \mathrm{F}}$ \\
\hline 1 & -152.72635679 & -152.72680212 & -97.74 & \\
2 & -152.72507096 & -152.72555341 & -105.89 & -8.15 \\
3 & -152.72460665 & -152.72511564 & -111.71 & -13.97 \\
4 & -152.72444819 & -152.72492466 & -104.57 & -6.83 \\
5 & -152.72363366 & -152.72412974 & -108.88 & -11.14 \\
6 & -152.72319016 & -152.72370876 & -113.82 & -16.08 \\
7 & -152.72201531 & -152.72273137 & -157.16 & -59.42 \\
8 & -152.71955119 & -152.72025968 & -155.50 & -57.76 \\
9 & -152.72192648 & -152.72276174 & -183.32 & -85.58 \\
10 & -152.72010319 & -152.72091276 & -177.68 & -79.94 \\
\hline
\end{tabular}

improvement is not as dramatic and the remaining discrepancies are much larger than the errors of the CC-pol-8s fit. This clearly suggests that the rigidity of the monomers in CC-pol-8s is responsible for most of the remaining discrepancies with the TLHVSQ values. Indeed, the addition of the monomer-flexibility corrections to the CC-pol-8s stationary point energies (second-to-last column) leads to barriers that are strikingly close to the benchmark values of Tschumper et al.: the RMSE is reduced from 46.6 to $6.4 \mathrm{~cm}^{-1}$. This agreement strongly suggests that the CC-pol-8s potential is within a few $\mathrm{cm}^{-1}$ from the rigid-monomer exact potential. The agreement found for CC-pol-8s can be contrasted with that achieved by two recent flexible-monomer potentials developed by Huang et al. ${ }^{10,11}$ and denoted as HBB0 and HBB, giving RMSEs of 34 and $33 \mathrm{~cm}^{-1}$, respectively. Since $\mathrm{HBB} 0$ and $\mathrm{HBB}$ already include monomer-flexibility effects, one can compare the barriers predicted by these potentials directly with the TLHVSQ barriers. Since the RMSEs of HBB0 and HBB with respect to the set of $a b$ initio points used to fit these potentials are very small $\left(0.006 \mathrm{kcal} \mathrm{mol}^{-1}\right.$ for energies up to $2000 \mathrm{~cm}^{-1}$ above the global minimum), the deviations of the barriers predicted by HBB 0 and HBB from the TLHVSQ ones are due (in contrast to the CC-pol potential) mainly to the basis sets used to compute $a b$ initio points in ref. 10 and 11 being smaller than those used to develop CC-pol. We also give in Table 7 the CC-pol barriers corrected for the monomer-flexibility effects. The RMSE in this case is $28 \mathrm{~cm}^{-1}\left(0.08 \mathrm{kcal} \mathrm{mol}^{-1}\right)$, a factor of two reduction compared to the rigid-monomer value. This discrepancy is consistent with the RMSE of CC-pol relative to $a b$ initio interaction energies for negative values of these energies.

Huang et al. ${ }^{10}$ give in Table 3 of their paper also barriers obtained with a rigid version of the HBB potential and the differences between fully-relaxed and rigid-monomer barriers are very small, below $2 \mathrm{~cm}^{-1}$ in all but three cases. These results may seem to contradict our findings. The reason for the smallness of monomer-flexibility effects found in ref. 10 is that 
Table 7 Interaction energies (in $\mathrm{cm}^{-1}$ ) at stationary points relative to the potential minimum (barriers) calculated with various potentials using the geometries optimized for individual potentials and compared to barriers obtained by Tschumper et al. ${ }^{13}$ Also the ab initio computed barriers at CC-pol-8s stationary points are listed

\begin{tabular}{|c|c|c|c|c|c|c|c|c|c|c|}
\hline No. & SAPT $-5 \mathrm{~s}^{a}$ & $\mathrm{HBB}^{b}$ & $\mathrm{HBB}^{c}$ & CC-pol ${ }^{d}$ & CC-pol-8s & $A b$ initio $^{e}$ & $\Delta E_{\mathrm{R} \rightarrow \mathrm{F}^{f}}$ & $5 \mathrm{~s}_{\mathrm{F}}^{g}$ & $8 \mathrm{~s}_{\mathrm{F}}^{h}$ & TLHVSQ \\
\hline 2 & & 164.1 & 161.4 & 187.9 & 190.5 & 189.2 & -8.2 & 179.8 & 182.3 & 181 \\
\hline 3 & 156 & 196.7 & 198.5 & 189.3 & 212.6 & 213.3 & -14.0 & 175.3 & 198.6 & 198 \\
\hline 4 & 185 & 244.7 & 244.0 & 211.1 & 250.0 & 246.7 & -6.8 & 204.3 & 243.2 & 245 \\
\hline 5 & & 328.6 & 329.3 & 330.5 & 342.4 & 341.0 & -11.1 & 319.3 & 331.3 & 333 \\
\hline 6 & 280 & 347.0 & 348.1 & 351.6 & 368.2 & 365.5 & -16.1 & 335.5 & 352.1 & 348 \\
\hline 7 & 659 & 601.4 & 603.0 & 746.4 & 702.4 & 704.5 & -59.4 & 687.0 & 643.0 & 634 \\
\hline 8 & 1244 & 1176.4 & 1181.8 & 1333.9 & 1293.6 & 1296.3 & -57.8 & 1276.1 & 1235.8 & 1249 \\
\hline 9 & 636 & 588.9 & 590.2 & 731.2 & 701.0 & 702.7 & -85.6 & 645.6 & 615.4 & 625 \\
\hline 10 & 897 & 897.1 & 898.3 & 1005.3 & 1027.0 & 1023.5 & -79.9 & 925.3 & 947.1 & 948 \\
\hline RMS & & 34.2 & 32.6 & 63.0 & 46.6 & 46.7 & & 27.9 & 6.4 & \\
\hline
\end{tabular}

${ }^{a}$ Ref. $6{ }^{b}$ Ref. 11, flexible-monomer potential. ${ }^{c}$ Ref. 10, flexible-monomer potential. ${ }^{d}$ Ref. $1 .{ }^{e}$ Calculated according to eqn (3) in ref. 1 at geometries predicted by the CC-pol-8s potential. ${ }^{f}$ Flexibility correction from Table $6 .{ }^{g}$ CC-pol prediction with added flexibility correction. ${ }^{h}$ CC-pol-8s prediction with added flexibility correction. ${ }^{i}$ Ref. $13 .{ }^{j}$ With respect to the TLHVSQ results.

their rigid monomers have the monomer equilibrium geometry rather than the vibrationally-averaged geometry applied by us. The 12D optimizations lead to monomer geometries deviating very little from the monomer equilibrium geometry, whereas the vibrationally-averaged geometry deviates quite significantly.

A similar approach to that described above can also be used to compute a monomer-flexibility correction to the depth of the global minimum. This can be achieved by applying eqn (7) to the interaction energies (rather than total dimer energies). Using the same level of theory and basis set as for the barriers, we obtain the ' $\mathrm{TF}$ ' and ' $\mathrm{TR}$ ' interaction energies equal to -4.853 and $-4.916 \mathrm{kcal} \mathrm{mol}^{-1}$, respectively. The resulting correction of $+0.063 \mathrm{kcal} \mathrm{mol}^{-1}$, when added to the CC-pol$8 \mathrm{~s}$ value of $-5.104 \mathrm{kcal} \mathrm{mol}^{-1}$ from Table 5 , gives the interaction energy of $-5.041 \mathrm{kcal} \mathrm{mol}^{-1}$, in a perfect agreement with the value of Tschumper et al..

The geometries of the stationary points predicted by the CC-pol-8s potential are contained in the ESI associated with this work. $\dagger$

\section{Vibration-rotation-tunneling spectrum of water dimer}

\section{A Computational methods}

It was demonstrated in previous work ${ }^{2,3,7,10,15,16,19-21}$ that the vibration-rotation-tunneling (VRT) levels of the water dimer measured by high resolution microwave and terahertz molecular beam spectroscopy ${ }^{22-30}$ provide an extremely critical test of the water pair potential. This is because the water dimer intermolecular potential surface has eight equivalent global minima separated by barriers low enough to enable the system to delocalize over these minima by quantum mechanical tunneling. The level splittings caused by this tunneling are very sensitive to the heights and shapes of the barriers. Also the low-frequency intermolecular vibrations, which have large amplitudes and are strongly anharmonic, probe the shape of the wells in the potential very sensitively. When this test was first applied ${ }^{19-21}$ to various available empirical and older $a b$ initio water potentials, it was concluded that none of these potentials was able to produce a realistic picture of the dimer VRT spectrum. More recently, it was demonstrated with the advent of the VRT(ASP-W) ${ }^{16}$ and VRT(MCY) ${ }^{31}$ potentials that the experimental VRT spectra could be used to improve $a b$ initio potentials by fitting a number of the parameters in their analytic representation to better reproduce the measured data. Later $a b$ initio potentials, such as SAPT $-5 s^{6}$ and SDFT, ${ }^{9}$ gave a fairly good reproduction of the dimer VRT spectrum without fitting any experimental data, although it was shown with the SAPT-5st potential ${ }^{7}$ that the use of such data could still improve the agreement with experiment. More recently, two purely $a b$ initio potentials, CC-pol ${ }^{1-3}$ and $\mathrm{HBB}^{10,11}$ published in 2006-2008, have reached such a high level of accuracy that the dimer VRT levels computed using these potentials are in even better agreement with experiment than the levels produced by the potentials improved by fitting the measured data. Here we apply the dimer VRT spectrum test to the CCpol-8s potential. Let us reiterate here that no experimental data have been utilized in the development of this potential and that it gives a better representation of the ab initio points than CC-pol only due to a more elaborate form of the fitting function.

In order to test a water pair potential by comparison with measured dimer VRT levels, it is required that these levels can be accurately calculated from a given global potential surface. Two methods which can do this are applied in this paper, the first one developed and implemented by Leforestier and coworkers, ${ }^{19,20}$ the second one by Groenenboom and coworkers. ${ }^{7}$ In both methods the water dimer geometry is described (for rigid monomers) by a set of Jacobi coordinates: the distance $R$ between the centers of mass of the monomers and the Euler angles $\alpha_{X}, \beta_{X}, \gamma_{X}$ describing the orientations of the water monomers $X=A, B$ with respect to a body-fixed frame on the dimer. The $z$-axis of this frame coincides with the vector $\boldsymbol{R}$ that points from the center of mass of monomer $A$ to that of monomer $B$. Note that the dimer geometry depends on the angles $\alpha_{A}$ and $\alpha_{B}$ only through the difference angle $\alpha=\alpha_{B}$ $\alpha_{A}$, so that the potential depends on the distance $R$ and five angles. These coordinates are the same as used in scattering calculations and the angular basis used in both methods is similar to the channel functions that one would use in scattering methods. It consists of a set of Wigner functions $D_{m_{X} k_{X}}^{\left(j_{X}\right)}\left(\alpha_{X}, \beta_{X}, \gamma_{X}\right)^{* 32}$ that describe the internal rotations of monomers $A$ and $B$, truncated at certain maximum values of $j_{A}$ 
and $j_{B}$. Most of the properties considered below have converged for the maximum $j_{X}$ value equal to 11 for the $\mathrm{H}_{2} \mathrm{O}$ dimer and 12 for the $\mathrm{D}_{2} \mathrm{O}$ dimer. For certain quantities such as the very small shifts of the levels caused by bifurcation tunneling, see below, we used a maximum of $j_{X}$ equal to 13 for the $\mathrm{H}_{2} \mathrm{O}$ dimer. The Wigner functions on the two monomers are Clebsch-Gordan coupled and multiplied by Wigner functions for the overall rotation of the complex with quantum numbers $J$, the total angular momentum of the complex, $M$, the projection of this angular momentum on a space-fixed $z$-axis, and $K$, the projection on the dimer $z$-axis. The quantum numbers $J$ and $M$ are exact quantum numbers. In the calculations by the method of Groenenboom et al., we neglected the off-diagonal Coriolis coupling between the internal angular momenta $\boldsymbol{j}_{A}, \boldsymbol{j}_{B}$, and the overall angular momentum $\boldsymbol{J}$, which makes also $K$ a good quantum number. The calculations by the method of Leforestier et al. were done both with and without inclusion of the off-diagonal Coriolis coupling. The water dimer is a near (prolate) symmetric top and the offdiagonal Coriolis coupling leads to a small asymmetry doubling of the water dimer levels for $|K|>0$.

The eight equivalent equilibrium structures of the water dimer are related to one another by interchanging the labels of the two hydrogen atoms on either one of the two $\mathrm{H}_{2} \mathrm{O}$ (or $\mathrm{D}_{2} \mathrm{O}$ ) monomers, and by an interchange of the two monomers. In other words, all interchanges of equivalent nuclei that do not break the chemical bonds within the two monomers are feasible, in the sense of Longuet-Higgins. ${ }^{33}$ These interchange operations, combined with inversion, generate the permutation-inversion (PI) symmetry group $G_{16}$ of the water dimer. In both methods the angular basis is adapted to the irreducible representations (irreps) $A_{1}^{ \pm}, B_{1}^{ \pm}, A_{2}^{ \pm}, B_{2}^{ \pm}$, and $E^{ \pm}$of this PI group. This simplifies the calculations considerably and is used in applying the spectral selection rules.

The two methods differ in the treatment of the radial coordinate $R$. In the method of Groenenboom et al., we used a primitive basis of 49 sinc-DVR (discrete variable representation) functions $\mathrm{s}^{34,35}$ for $R$ ranging from 4 to $9 a_{0}$. This basis was contracted to three radial basis functions obtained by using a one-dimensional radial cut through the equilibrium geometry in the six-dimensional potential surface, see ref. 7. Such a basis, although small, gives well-converged results. With the enlargement from three to four radial basis functions, the VRT levels hardly changed. ${ }^{3}$ Also extending the outer boundary of $R$ from 9 to $10 a_{0}$ gave practically no change. In the method of Leforestier et al., we started from a sine-DVR with a primitive $R$-grid of 30 points in the range from 4 to $10 a_{0}$. Then we applied the potential-optimized contraction scheme of Harris et al. ${ }^{36}$ with the contracted grid comprising 9 points. Also this scheme was tested and it was found that the number of points used produces sufficiently well converged VRT levels.

Although the analytical angular basis is the same in the two methods, there is an essential difference between them in the treatment of the angular coordinates. The method of Leforestier et al. is a pseudo-spectral method that uses, in addition to the analytical basis of Wigner $D$-functions, an angular grid basis of numerical quadrature points appropriate for these functions. ${ }^{37}$ In the Lanczos algorithm used to iteratively determine a set of the lowest eigenvalues of the Hamiltonian matrix, one should multiply a set of trial eigenvectors by this matrix in each iteration. The potential is diagonal in the grid basis and the diagonal potential matrix elements are the values of the potential in the grid points. The trial eigenvectors in the analytical basis are transformed to the grid basis, multiplied with the potential in this basis, and then transformed back to the analytical basis. The kinetic energy matrix is evaluated in the analytical angular basis and multiplied with the trial vectors in this basis. This makes this method extremely economical both in the use of storage and in computer time. The method of Groenenboom et al. uses the Davidson algorithm to iteratively determine a set of lowest energy levels; also this algorithm requires the multiplication of trial eigenvectors by the Hamiltonian matrix. All non-zero elements of this matrix are here evaluated in the analytical basis. The potential is expanded in the same coupled set of Wigner $D$-functions as used in the angular basis, which is simplified, however, because the potential is a scalar function of $A_{1}^{+}$symmetry. For each of the radial grid points, the $R$-dependent coefficients in this expansion are evaluated by numerical integration over the angles; the angular quadrature grid is of the same type as the grid that the method of Leforestier et al. uses as the angular grid basis. Subsequently these coefficients are transformed to the much smaller contracted radial basis. With the use of such an expansion for the potential, all angular integrals can be evaluated analytically in terms of $3-j$ and $9-j$ symbols. ${ }^{32}$

Before discussing the results, we define some important parameters that characterize the spectra of the water dimer. Since the energy levels can be grouped into rotational ladders with rungs for increasing values of $J \geq|K|$ and the water dimer is a near-symmetric top, it is customary to represent each ladder by the formula

$$
E(J, K)=o(K)+\frac{(B+C)}{2}\left[J(J+1)-K^{2}\right],
$$

where $o(K)$ is the origin for a given value of $|K|$ and $(B+C) / 2$ is the end-over-end rotational constant of the dimer. The same formula with some additional terms to include the very small asymmetry effects was used to represent the levels extracted from the experimental spectra. ${ }^{27,30}$ We obtained the origins $o(K)$ for $|K|=0$ and 1 by applying this formula to the levels calculated with $J=|K|$. Each $J, K$ level is split by tunneling, however, see Fig. 1 and 2. Acceptor tunneling causes the largest splittings: for $K=0$ and even $J$ each level is split into a pair of levels with $A_{1}^{+}$and $B_{1}^{+}$symmetry and a pair of levels with $A_{2}^{-}$ and $B_{2}^{-}$symmetry. For odd $J$ and $K=0$, the corresponding levels have $B_{1}^{-}$and $A_{1}^{-}$symmetry and $B_{2}^{+}$and $A_{2}^{+}$ symmetry, respectively, while for $|K|>0$ they occur in pairs of $A_{1}^{ \pm}, B_{1}^{\mp}$ levels and $A_{2}^{\mp}, B_{2}^{ \pm}$levels that are very slightly separated in energy by asymmetry doubling (caused by offdiagonal Coriolis coupling terms in our calculations). Moreover, there are levels of $E^{ \pm}$symmetry which are always between the $A_{1}^{ \pm}$and $B_{1}^{ \pm}$levels and between the $A_{2}^{ \pm}$and $B_{2}^{ \pm}$ levels of the same \pm parity. These are sometimes labeled $E_{1}^{ \pm}$ and $E_{2}^{ \pm}$, although the latter labels have no strict meaning. The origins $o_{1}(K)$ and $o_{2}(K)$ are defined separately for the levels of $A_{1}^{ \pm}, B_{1}^{ \pm}$symmetry and the levels of $A_{2}^{ \pm}, B_{2}^{ \pm}$symmetry, respectively, by averaging the energies of these levels over the smaller splittings between the $A_{1}^{ \pm}$and $B_{1}^{ \pm}$levels and between the $A_{2}^{\mp}$ 


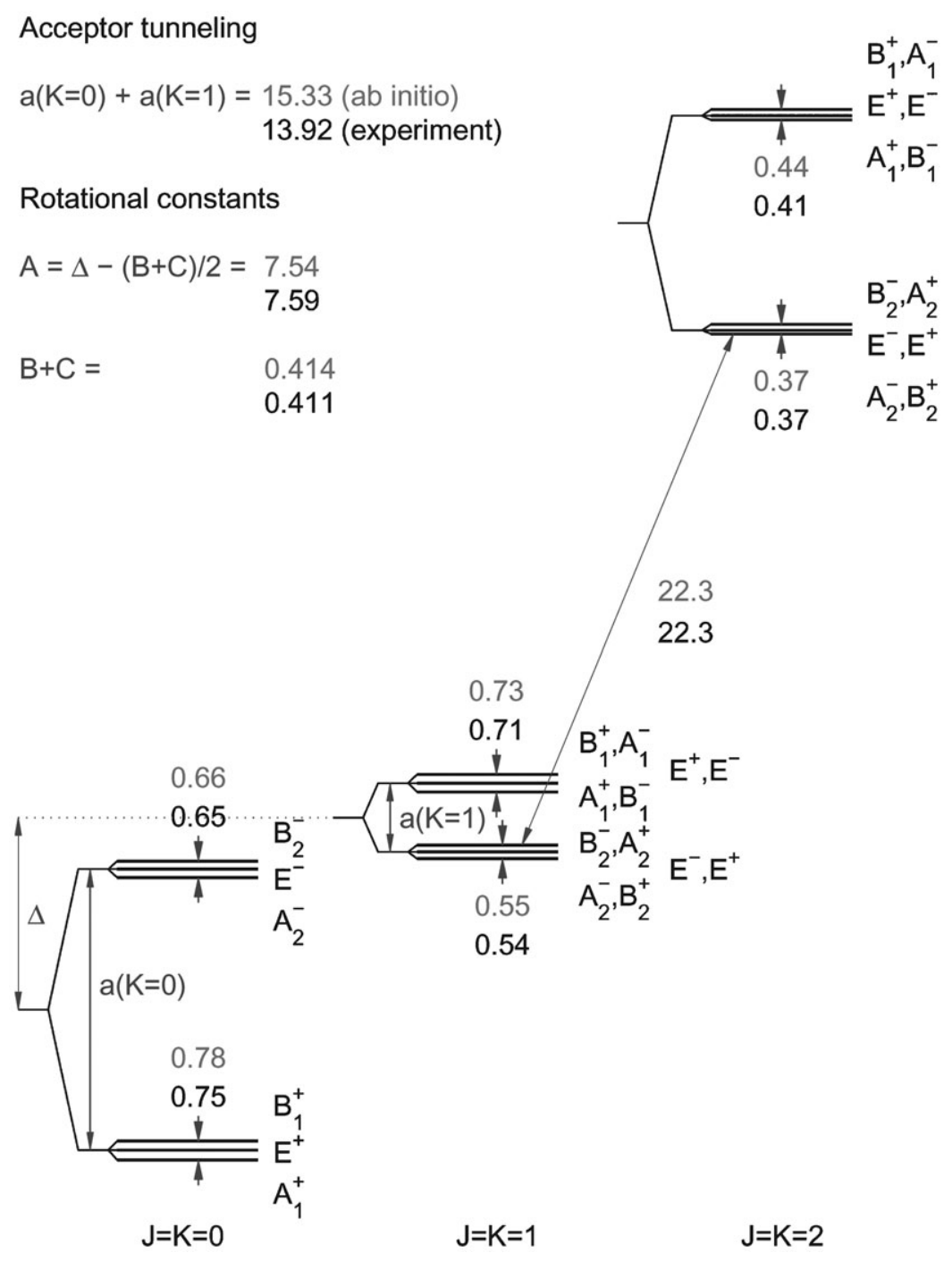

Fig. 1 Ground state VRT levels of the $\mathrm{H}_{2} \mathrm{O}$ dimer $\left(\right.$ in $\mathrm{cm}^{-1}$ ) from converged calculations with the CC-pol-8s ab initio potential, in comparison with experimental data ${ }^{23,24,28}$ (lower numbers). The energies are drawn to scale (using experimental values).

and $B_{2}^{\mp}$ levels. The latter splittings are due to interchange tunneling and are represented by the parameters $i_{1}$, which is the energy difference between a pair of $B_{1}^{ \pm}$and $A_{1}^{ \pm}$levels, and $i_{2}$, which is the energy difference between a pair of $B_{2}^{\mp}$ and $A_{2}^{\mp}$ levels. The acceptor tunneling splitting $a(K)$ can be extracted from the (calculated or measured) spectra as the energy difference between the origins $o_{2}(K)$ and $o_{1}(K)$.

Other quantities discussed are the end-over-end rotational constant $(B+C) / 2$ and the much larger rotational constant $A$ for rotation about the prolate axis (nearly coinciding with the vector $\boldsymbol{R}$ ). The value of $B+C$ for $K=0$ was obtained from our calculations as the difference between levels with $J=1$ and $J=0$ and the value of $B+C$ for $|K|=1$ as half the difference between levels with $J=2$ and $J=1$. These constants are only slightly different (on the order of 0.001 $\mathrm{cm}^{-1}$ ) for the states of different symmetry split by tunneling; we present symmetry-averaged values. Then, from the usual prolate near-symmetric top expression

$$
E(J, K)=E(0,0)+\frac{(B+C)}{2}\left[J(J+1)-K^{2}\right]+A K^{2}
$$

and the definition of the origins in eqn (8), it follows that $A$ is simply the difference between the $|K|=1$ and $K=0$ origins. Here we applied this formula to the averaged origins $\left[o_{1}(K)+o_{2}(K)\right] / 2$. Note that for $\left(\mathrm{H}_{2} \mathrm{O}\right)_{2}$ two different experimental values can be found in the literature: $A=7.44 \mathrm{~cm}^{-1}$ extracted from the microwave spectrum ${ }^{23}$ and $A=7.59 \mathrm{~cm}^{-1}$ from terahertz spectra. ${ }^{28,30}$

The shifts $b_{1}$ and $b_{2}$ of the energies of the $E^{ \pm}$levels with respect to the average energies of the $A_{1}^{ \pm}$and $B_{1}^{ \pm}$levels and of the $A_{2}^{ \pm}$and $B_{2}^{ \pm}$levels are due to bifurcation tunneling. These shifts are very small since the energy barrier associated with this process is relatively high. For $J=K=0$, the shifts are defined by

$$
\begin{aligned}
& b_{1}^{(0)}=\left[E\left(E_{1}^{+}\right)-\left[E\left(A_{1}^{+}\right)+E\left(B_{1}^{+}\right)\right] / 2\right] / 2 \\
& b_{2}^{(0)}=\left[E\left(E_{2}^{-}\right)-\left[E\left(A_{2}^{-}\right)+E\left(B_{2}^{-}\right)\right] / 2\right] / 2,
\end{aligned}
$$

and for $J=|K|=1$ by

$$
\begin{aligned}
b_{1}^{(1)}= & {\left[E\left(E_{1}^{+}\right)-\left[E\left(A_{1}^{+}\right)+E\left(B_{1}^{+}\right)\right] / 2+E\left(E_{1}^{-}\right)\right.} \\
& \left.-\left[E\left(B_{1}^{-}\right)+E\left(A_{1}^{-}\right)\right] / 2\right] / 4,
\end{aligned}
$$




\section{Acceptor tunneling}

$\begin{array}{llll}\mathrm{a}(\mathrm{K}=0) & \mathrm{a}(\mathrm{K}=1) & \mathrm{a}(\mathrm{K}=2) & \\ 2.04 & 0.64 & 1.48 & \text { (ab initio) } \\ 1.77 & 0.62 & 1.31 & \text { (experiment) }\end{array}$

\section{Rotational constants}

$$
\begin{array}{lr}
\mathrm{A}=\Delta-(\mathrm{B}+\mathrm{C}) / 2= & 4.20 \\
& 4.17 \\
\mathrm{~B}+\mathrm{C}= & 0.361
\end{array}
$$
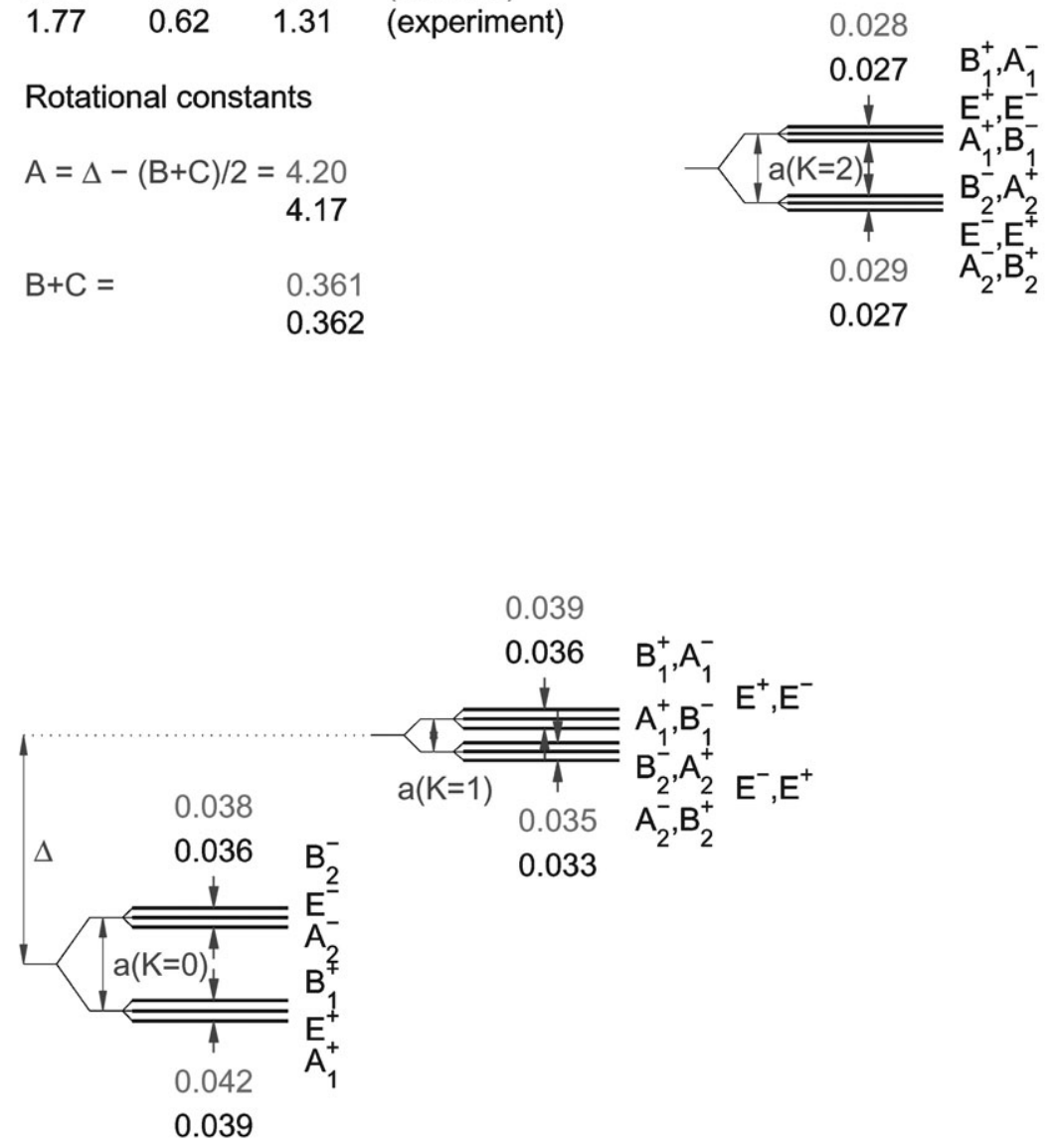

$\mathrm{J}=\mathrm{K}=0$

$\mathrm{J}=\mathrm{K}=1$

$\mathrm{J}=\mathrm{K}=2$

Fig. 2 Ground state VRT levels of the $\mathrm{D}_{2} \mathrm{O}$ dimer $\left(i n \mathrm{~cm}^{-1}\right.$ ) calculated from the CC-pol-8s $a b$ initio potential, in comparison with experimental data $^{22,25-27}$ (lower numbers). The energies are drawn to scale (using experimental values), except for the small interchange splittings which are enlarged by a factor of 10 .

$$
\begin{aligned}
b_{2}^{(1)}= & {\left[E\left(E_{2}^{-}\right)-\left[E\left(A_{2}^{-}\right)+E\left(B_{2}^{-}\right)\right] / 2+E\left(E_{2}^{+}\right)\right.} \\
& \left.-\left[E\left(B_{2}^{+}\right)+E\left(A_{2}^{+}\right)\right] / 2\right] / 4,
\end{aligned}
$$

where $E_{1}^{ \pm}$denotes the $E^{ \pm}$levels situated between the $A_{1}^{ \pm}$and $B_{1}^{ \pm}$levels and $E_{2}^{ \pm}$the $E^{ \pm}$levels situated between the $A_{2}^{ \pm}$and $B_{2}^{ \pm}$levels. From the measurements ${ }^{29}$ one extracts the differences $b_{1}^{(01)}=\left[b_{1}^{(0)}-b_{1}^{(1)}\right] / 2$ and $b_{2}^{(01)}=\left[b_{2}^{(0)}-b_{2}^{(1)}\right] / 2$.

\section{B Results and discussion}

Most of the properties of the states with $K=0$ and $|K|=1$ calculated on the CC-pol-8s potential are listed and compared with the available experimental data in Tables 8 and 9 for the $\mathrm{H}_{2} \mathrm{O}$ dimer and $\mathrm{D}_{2} \mathrm{O}$ dimer, respectively. The calculated and measured ground state tunneling levels of the $\mathrm{H}_{2} \mathrm{O}$ and $\mathrm{D}_{2} \mathrm{O}$ dimers are displayed in Fig. 1 and 2. The quantity $\Delta$ shown in Fig. 1 and 2 and used to compute the rotational constant $A$ is the energy difference between the levels with $J=|K|=1$ and those with $J=K=0$, averaged over all symmetries $A_{1}^{+}, B_{1}^{+}$,
$A_{2}^{-}, B_{2}^{-}$. The levels [origins $o_{1}(K)$ and $o_{2}(K)$ for $K=0,1$ ] corresponding to the intermolecular vibrations of the $\mathrm{H}_{2} \mathrm{O}$ dimer and the $\mathrm{D}_{2} \mathrm{O}$ dimer are shown in Fig. 3 and 4, respectively. The assignment of these intermolecular vibrations was discussed in ref. 39, on the basis of the VRT wave functions calculated on the SAPT-5st potential surface. Note that the mode that was assigned to the in-plane bend fundamental in the earlier experimental and theoretical papers, on the basis of approximate harmonic oscillator calculations, is actually the donor torsion overtone.

The dissociation energies computed with CC-pol-8s are $D_{0}$ $=1094 \mathrm{~cm}^{-1}$ for the $\mathrm{H}_{2} \mathrm{O}$ dimer and $D_{0}=1241 \mathrm{~cm}^{-1}$ for the $\mathrm{D}_{2} \mathrm{O}$ dimer. These energies are somewhat smaller than the corresponding values from CC-pol, which were $D_{0}=1111$ $\mathrm{cm}^{-1}$ for the $\mathrm{H}_{2} \mathrm{O}$ dimer and $1255 \mathrm{~cm}^{-1}$ for the $\mathrm{D}_{2} \mathrm{O}$ dimer. The well depths of CC-pol-8s and CC-pol are very similar: $D_{e}=5.104 \mathrm{kcal} \mathrm{mol}^{-1}=1785 \mathrm{~cm}^{-1}$ and $5.097 \mathrm{kcal}$ $\mathrm{mol}^{-1}=1783 \mathrm{~cm}^{-1}$, respectively. Obviously, the zero-point 
Table 8 VRT levels, tunneling splittings, and rotational constants $\left(\right.$ in $\left.\mathrm{cm}^{-1}\right)$ of $\left(\mathrm{H}_{2} \mathrm{O}\right)_{2}$ calculated from the CC-pol- 8 s potential. The numbers in parentheses are experimental values from ref. 23, 24, 28-30. The assignment of the intermolecular vibrations is given in ref. 39 . When it deviates from the earlier assignment according to ref. 28, the latter is added in square brackets. The energy zero corresponds to $D_{0}=1093.54 \mathrm{~cm}^{-1}$

\begin{tabular}{|c|c|c|c|c|c|c|c|}
\hline & $o_{1}$ & $o_{2}{ }^{a}$ & $a$ & $i_{1}$ & $i_{2}$ & $B+C$ & $A$ \\
\hline \multicolumn{8}{|c|}{ Ground state $\left(A^{\prime}\right)$} \\
\hline$K=0$ & $\begin{array}{c}0 \\
(0)\end{array}$ & $\begin{array}{c}12.39 \\
(11.18)\end{array}$ & $\begin{array}{l}12.39 \\
\left({ }^{b}\right)\end{array}$ & $\begin{array}{c}0.783 \\
(0.752)\end{array}$ & $\begin{array}{c}0.664 \\
(0.651)\end{array}$ & $\begin{array}{c}0.4137 \\
(0.4112)\end{array}$ & \\
\hline$K=1$ & $\begin{array}{c}15.20 \\
(14.39)\end{array}$ & $\begin{array}{c}12.26 \\
(11.66)\end{array}$ & $\begin{array}{l}2.94 \\
\left({ }^{b}\right)\end{array}$ & $\begin{array}{c}0.732 \\
(0.705)\end{array}$ & $\begin{array}{c}0.555 \\
(0.541)\end{array}$ & $\begin{array}{c}0.4067 \\
(0.4108)\end{array}$ & $\begin{array}{l}7.54 \\
\left(7.44 / 7.59^{c}\right)\end{array}$ \\
\hline \multicolumn{8}{|c|}{ Donor torsion $\left(A^{\prime \prime}\right)$} \\
\hline$K=0$ & 113.30 & $\begin{array}{c}61.65 \\
(64.52)\end{array}$ & 51.65 & 6.365 & $\begin{array}{c}2.721 \\
(2.54)\end{array}$ & 0.4132 & \\
\hline$K=1$ & $\begin{array}{c}86.23 \\
(87.75)\end{array}$ & 92.17 & 5.93 & $\begin{array}{c}1.390 \\
(1.110)\end{array}$ & 3.693 & $\begin{array}{c}0.4096 \\
(0.4083)\end{array}$ & 1.73 \\
\hline \multicolumn{8}{|c|}{ Acceptor wag $\left(A^{\prime}\right)$} \\
\hline$K=0$ & $\begin{array}{c}109.51 \\
(107.93)\end{array}$ & $\begin{array}{c}109.78 \\
(108.89)\end{array}$ & $\begin{array}{c}0.28 \\
(0.96)\end{array}$ & $\begin{array}{c}3.779 \\
(2.951)\end{array}$ & $\begin{array}{c}0.031 \\
(0.017)\end{array}$ & $\begin{array}{c}0.4106 \\
(0.4094)\end{array}$ & \\
\hline$K=1$ & $\begin{array}{c}109.59 \\
(109.98)\end{array}$ & $\begin{array}{c}123.83 \\
(123.56)\end{array}$ & $\begin{array}{c}14.24 \\
(13.58)\end{array}$ & $\begin{array}{c}5.603 \\
(5.238)\end{array}$ & $\begin{array}{c}4.028 \\
(3.412)\end{array}$ & $\begin{array}{c}0.4085 \\
(0.4122)\end{array}$ & $\begin{array}{c}7.06 \\
(8.08)\end{array}$ \\
\hline \multicolumn{8}{|c|}{ Acceptor twist $\left(A^{\prime \prime}\right)$} \\
\hline$K=0$ & 132.14 & $\begin{array}{c}118.10 \\
(120.19)\end{array}$ & 14.04 & 0.970 & $\begin{array}{c}9.717 \\
(9.393)\end{array}$ & $\begin{array}{c}0.4110 \\
(0.4138)\end{array}$ & \\
\hline$K=1$ & 143.22 & 137.25 & 5.97 & 4.632 & 5.405 & 0.4089 & 15.12 \\
\hline \multicolumn{8}{|c|}{ Donor torsion overtone $\left(A^{\prime}\right)$ [in-plane bend] } \\
\hline$K=0$ & 128.24 & $\begin{array}{c}153.44 \\
(153.62)\end{array}$ & 25.20 & 10.712 & $\begin{array}{c}1.904 \\
(1.877)\end{array}$ & 0.4082 & \\
\hline $\begin{array}{l}K=1 \\
\text { Stretch }\left(A^{\prime}\right)\end{array}$ & 155.61 & 152.17 & 3.43 & 2.457 & 3.855 & 0.4010 & 13.05 \\
\hline$K=0$ & 147.65 & 185.73 & 38.08 & 2.470 & 19.460 & $0.4064^{d}$ & \\
\hline
\end{tabular}

${ }^{a}$ Since the experimental values of $o_{2}$ were given relative to the ground state value of $o_{2}$, we added the ground state acceptor splitting $a(K=0)=$ $11.18 \mathrm{~cm}^{-1}$ estimated in ref. 7 to all experimental values. ${ }^{b}$ Acceptor splitting $a(K=0)+a(K=1)=15.33 \mathrm{~cm}^{-1}$ (calculated) and $13.92 \mathrm{~cm}^{-1}$ (experimental). ${ }^{c}$ From data in ref. 23 and 28, respectively. ${ }^{d}$ The $B+C$ value for $A_{2}^{-}$symmetry was excluded from this average value, because the corresponding $K=0, J=1$ level of $B_{2}^{+}$symmetry is strongly perturbed by Coriolis interaction with the $|K|=1, J=1$ level of the same symmetry and nearly the same energy (about $-917.2 \mathrm{~cm}^{-1}$ ). Also the asymmetry doubling of the $A_{2}^{-}$and $B_{2}^{+}$levels with $|K|=1$ is anomalously large because of this resonance.

energy of intermolecular vibration (and hindered rotation) in the water dimer is somewhat higher for CC-pol-8s than for CC-pol. Experimentally, $D_{0}$ has not been accurately determined, so our results provide the most reliable value of this quantity.

When comparing Tables 8 and 9 with Tables I and II of ref. 3 , we observe that the results from CC-pol-8s agree substantially better with experiment than those of CC-pol. This is quantified by the percentage errors in Table 10. By examining the items in Tables 8 and 9, one notices that the improvement is very systematic: each individual quantity, practically without exception, agrees better with experiment for CC-pol-8s than for CC-pol. This is very satisfactory, because one can see in Table 10 that the results from CC-pol already agreed well with the measured high-resolution spectra, much better than those of the recent TTM2.1 ab initio potential, ${ }^{40}$ for example, and even better than those of VRT(ASP-W)III potential fitted to these spectra. The only potential that agreed about equally well with experiment as CC-pol, for some quantities even better, is the recent $a b$ initio potential HBB, $c f$. Tables IV and $\mathrm{V}$ of ref. 10. In the discussion below, we will comment on the results from the latter potential in more detail. It is remarkable, for example, that CC-pol-8s predicts the frequencies of the strongly anharmonic intermolecular vibrations with discrepancies of only $2.3 \%$ for the $\mathrm{H}_{2} \mathrm{O}$ dimer and only $1.7 \%$ for the $\mathrm{D}_{2} \mathrm{O}$ dimer; note that more experimental data are available in the latter case. In the comparison in Table 10, we included only the band origins of the vibrationally excited states with $K=0$, but one can see in Tables 8 and 9 that the agreement is equally good for $|K|=1$. Even the acceptor and interchange tunneling splittings in these vibrationally excited states agree well with experiment for CC-pol-8s, whilst those from CC-pol ${ }^{3}$ still deviated substantially. This improvement is almost completely due to the more accurate fit of the ab initio data points, see section 2 , the effect of the small correction of the $a b$ initio data points is nearly negligible. We can substantiate this statement because we also computed the VRT levels of the $\mathrm{H}_{2} \mathrm{O}$ and $\mathrm{D}_{2} \mathrm{O}$ dimers from a similarly good fit to the uncorrected $a b$ initio points and obtained results that are very similar to the results presented here. Also the fact that some of the results from the HBB potential agreed better with experiment than those from CC-pol was due to a more accurate numerical fit of the $a b$ initio data points in the case of $\mathrm{HBB}$ potential. The fit of CC-pol was constrained to obey the correct long-range behavior and its analytic form was intentionally kept relatively simple in view of its application in liquid water simulations. This is why the fit of the ab initio data points by CC-pol was numerically less accurate than that of $\mathrm{HBB}$, whereas the CC-pol-8s fit, which has the same correct long-range behavior as CC-pol, is about equally accurate as the $\mathrm{HBB}$ fit in terms of reproducing $a b$ initio points (the RMSE of HBB for the first $2000 \mathrm{~cm}^{-1}$ is $0.006 \mathrm{kcal} \mathrm{mol}^{-1}$ ). As discussed earlier, the points are more accurate in the case of CC-pol-8s. 
Table 9 VRT levels, tunneling splittings, and rotational constants $\left(\right.$ in $\left.\mathrm{cm}^{-1}\right)$ of $\left(\mathrm{D}_{2} \mathrm{O}\right)_{2}$ calculated from the CC-pol-8s potential. The numbers in parentheses are experimental values from ref. 22, 25-27, 29 and 30 . The energy zero corresponds to $D_{0}=1241.08 \mathrm{~cm}^{-1}$

\begin{tabular}{|c|c|c|c|c|c|c|c|}
\hline & $o_{1}$ & $\mathrm{o}_{2}{ }^{a}$ & $a$ & $i_{1}$ & $i_{2}$ & $B+C$ & $A$ \\
\hline \multicolumn{8}{|c|}{ Ground state $\left(A^{\prime}\right)$} \\
\hline \multirow[t]{2}{*}{$K=0$} & 0 & 2.04 & 2.04 & 0.0422 & 0.0385 & 0.3610 & \\
\hline & $(0)$ & $(1.77)$ & $(1.77)$ & $(0.0391)$ & $(0.0361)$ & $(0.3622)$ & \\
\hline \multirow[t]{2}{*}{$K=1$} & 5.54 & 4.89 & 0.64 & 0.0386 & 0.0353 & 0.3609 & 4.20 \\
\hline & $(5.36)$ & $(4.74)$ & $(0.62)$ & $(0.0359)$ & $(0.0331)$ & $(0.3621)$ & $(4.17)$ \\
\hline \multicolumn{8}{|c|}{ Donor torsion $\left(A^{\prime \prime}\right)$} \\
\hline \multirow[t]{2}{*}{$K=0$} & 74.41 & 57.54 & 16.87 & 0.374 & 0.223 & 0.3612 & \\
\hline & (75.38) & (59.59) & $(15.81)$ & $(0.328)$ & $(0.203)$ & $(0.3622)$ & \\
\hline \multirow[t]{2}{*}{$K=1$} & 66.60 & 70.24 & 3.64 & 0.149 & 0.283 & 0.3589 & 2.45 \\
\hline & $(68.27)$ & $(71.81)$ & $(3.54)$ & $(0.132)$ & $(0.257)$ & $(0.3600)$ & $(2.56)$ \\
\hline \multicolumn{8}{|c|}{ Acceptor wag $\left(A^{\prime}\right)$} \\
\hline \multirow[t]{2}{*}{$K=0$} & 82.35 & 84.29 & 1.94 & 0.141 & 0.124 & 0.3590 & \\
\hline & (82.64) & $(84.40)$ & $(1.77)$ & $(0.131)$ & $(0.112)$ & $(0.3603)$ & \\
\hline \multirow[t]{2}{*}{$K=1$} & 84.94 & 89.62 & 4.68 & 0.444 & 0.196 & 0.3619 & 3.96 \\
\hline & $(85.57)$ & $(89.56)$ & $(4.00)$ & $(0.398)$ & $(0.168)$ & $(0.3592)$ & $(4.05)$ \\
\hline \multicolumn{8}{|c|}{ Acceptor twist $\left(A^{\prime \prime}\right)$} \\
\hline \multirow[t]{2}{*}{$K=0$} & 92.22 & 88.96 & 3.26 & 0.412 & 0.817 & 0.3627 & \\
\hline & $(92.91)$ & $(90.37)$ & $(2.54)$ & $(0.432)$ & $(0.443)$ & $(0.3665)$ & \\
\hline$K=1$ & 98.17 & 95.24 & 2.92 & 0.476 & 0.615 & 0.3649 & 6.12 \\
\hline \multicolumn{8}{|c|}{ Donor torsion overtone $\left(A^{\prime}\right)$ [in-plane bend] } \\
\hline$K=0$ & $\begin{array}{l}102.22 \\
(104.24)\end{array}$ & 133.87 & 31.65 & $\begin{array}{c}0.936 \\
(0.783)\end{array}$ & 2.000 & $\begin{array}{c}0.3660 \\
(0.3632)\end{array}$ & \\
\hline$K=1$ & 124.63 & 117.19 & 7.44 & 0.683 & 0.023 & 0.3604 & 2.87 \\
\hline \multicolumn{8}{|c|}{ Donor torsion + acceptor wag combination $\left(A^{\prime \prime}\right)$} \\
\hline$K=0$ & 133.45 & 138.70 & 5.24 & 0.075 & 1.145 & 0.3611 & \\
\hline \multicolumn{8}{|c|}{ Stretch $\left(A^{\prime}\right)$} \\
\hline$K=0$ & 135.64 & 144.18 & 8.55 & 3.451 & 1.701 & 0.3785 & \\
\hline
\end{tabular}

The only quantity that still deviates substantially from the experimental value is the acceptor tunneling splitting $a(K)$, although the error is only half as large with CC-pol-8s as the error in this quantity with CC-pol and HBB (about one third for $\mathrm{D}_{2} \mathrm{O}$ dimer). Calculations with the 12-dimensional (12D) $\mathrm{HBB}^{41}$ and $\mathrm{SAPT}^{42}$ potentials with both flexible and rigid monomers show that $a(K)$ is overestimated by the use of rigid monomers and that the value of $a(K)$ from full 12D calculations of the VRT levels agrees well with experiment. Thus, we conclude that the overestimate of $a(K)$ by CC-pol-8s is mainly due to its use of rigid monomers. The error produced by $\mathrm{CC}$ pol-8s is much smaller than that produced by CC-pol due to the better fit. The fact that it is also smaller than one produced by $\mathrm{HBB}$ when the latter was used in $6 \mathrm{D}$ calculations, i.e., with rigid monomers ${ }^{10}$ is partly due to the use of monomers in their equilibrium geometry in the $\mathrm{HBB}$ case and monomers in their vibrationally averaged ground state geometry in the CC-pol-8s case. It was demonstrated in ref. 43 that the use of vibrationally averaged monomer geometries gives results that are closer to the "exact" flexible monomer results. The other reason is the better quality of the ab initio points used to fit CC-pol-8s. The 12D calculations with $\mathrm{HBB}^{41}$ also show that all other calculated quantities: interchange tunneling splittings, rotational constants, vibrational frequencies, are only very slightly affected by the use of rigid monomers; 6D and 12D calculations give practically the same results. Thus, we may conclude that the excellent agreement of the CC-pol-8s values with experiment for all quantities except $a(K)$ will remain equally good if we would "unfreeze" the monomer geometries.
The values presented in Table 8 for the $\mathrm{H}_{2} \mathrm{O}$ dimer and in Table 9 for the $\mathrm{D}_{2} \mathrm{O}$ dimer were computed with the method of Leforestier et al. The corresponding values obtained by the method of Groenenboom et al. differ only very slightly from these values, see Table 10, so we have not included them in Tables 8 and 9. This agreement between two rather different methods gives an important validation for both methods and also allows to estimate the accuracy of the computed values. From various tests of the parameters on which the two methods depend, such as the radial basis, the angular grid in the method of Leforestier et al., the angular expansion of the potential in the method of Groenenboom et al., etc., it could be established that the remaining very small differences between the two methods are mostly due to the truncation of the latter expansion at spherical harmonics with maximum values of $L_{A}$ and $L_{B}$ equal to 8 .

A more remarkable difference concerns the end-over-end rotational constants $B+C$. When we compare this quantity with the neglect of the off-diagonal Coriolis coupling, i.e., with the assumption that $K$ is a good quantum number, the values from the two methods agree very closely. Previously, we assumed that we could neglect this coupling because we believed that its main effect is to give the small asymmetry doublet splittings of the levels with $|K|>0$. When Coriolis coupling was fully included, we found, indeed, in nice agreement with experiment, that the asymmetry doublet splittings of the levels with (mainly) $|K|=1$ are very small, on the order of $0.01 \mathrm{~cm}^{-1}$ at most, but more typically $0.001 \mathrm{~cm}^{-1}$ or less for $J=1$. For $J=2$, the splittings are about three times as large, as one expects for a Coriolis coupling effect proportional to 


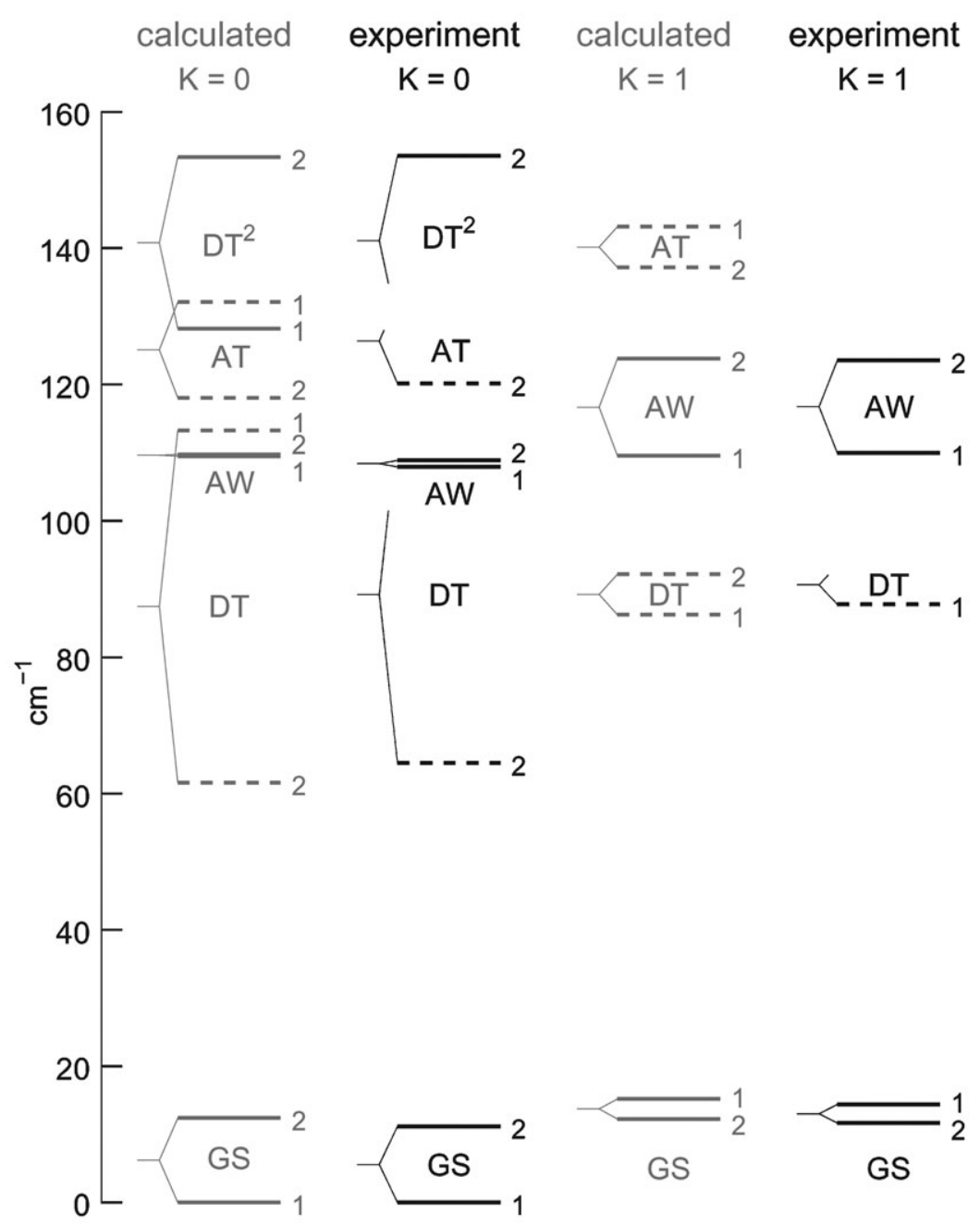

Fig. 3 VRT levels of the $\mathrm{H}_{2} \mathrm{O}$ dimer corresponding to the intermolecular vibrations, calculated from the CC-pol-8s potential, in comparison with experimental data. ${ }^{28,38}$ The levels 1 and 2 are the origins, $o_{1}$ and $o_{2}$, of the $A_{1}, E_{1}, B_{1}$ and $A_{2}, E_{2}, B_{2}$ levels, respectively. The abbreviations GS, DT, $\mathrm{AW}$, and AT denote the ground state $\left(A^{\prime}\right)$, donor torsion $\left(A^{\prime \prime}\right)$, acceptor wag $\left(A^{\prime}\right)$, and acceptor twist $\left(A^{\prime \prime}\right)$ modes, following ref. 28 and 39 . Solid lines refer to $A^{\prime}$ symmetry, dashed lines to $A^{\prime \prime}$ symmetry, with respect to the point group $C_{\mathrm{s}}$ of the equilibrium structure. Experimental levels not shown have not been measured to date.

$J(J+1)$. For the (mainly) $|K|=2$ levels, the splittings are still smaller by a few orders of magnitude, because such splittings originate from an indirect coupling through the $|K|=1$ levels. The rotational constants and tunneling splittings that we extracted from the levels with $|K|>0$ are very similar for the two opposite parity components of the doublets; the values presented are averages. What we had not expected, however, was that the off-diagonal Coriolis coupling has an effect on the values of $B+C$ which becomes significant now that we have reached such a high level of accuracy in the calculations of the VRT levels with CC-pol-8s. The values of $B+C$ for the $\mathrm{H}_{2} \mathrm{O}$ dimer decrease by about $0.01 \mathrm{~cm}^{-1}$ due to the inclusion of the off-diagonal Coriolis coupling. For the $\mathrm{D}_{2} \mathrm{O}$ dimer, where also the asymmetry doubling is larger because of the larger mass of the $\mathrm{D}$ atoms that protrude from the dimer axis, the $B+C$ values decrease even more: by almost $0.02 \mathrm{~cm}^{-1}$. The values computed from CC-pol-8s with the off-diagonal Coriolis coupling neglected are very similar to the CC-pol values, which were about $0.01 \mathrm{~cm}^{-1}$ too large with respect to the measured values for $\mathrm{H}_{2} \mathrm{O}$ dimer. With the inclusion of the offdiagonal Coriolis coupling, this discrepancy is removed and we obtain excellent agreement with experiment for $B+C$. We must also conclude now that the excellent agreement of the $B+C$ values from the $\mathrm{HBB}$ potential ${ }^{10}$ with experiment is partly fortuitous. If Coriolis couplings were fully included, the HHB values would be too small, in agreement with the binding energy $D_{e}$ of this potential being too small and the equilibrium distance being slightly too large.

The accuracy of the CC-pol-8s results discussed so far was so high that we found it worthwhile to consider also the small shifts of the $E^{ \pm}$levels with respect to the average energies of the $A_{1}^{ \pm}$and $B_{1}^{ \pm}$levels and those of the $A_{2}^{\mp}$ and $B_{2}^{\mp}$ levels. These shifts are caused by bifurcation tunneling and are defined by eqns (10) and (11). The shifts were calculated only for the $\mathrm{H}_{2} \mathrm{O}$ dimer, because such shifts are still smaller by about a factor of 100 for the $\mathrm{D}_{2} \mathrm{O}$ dimer and could not be converged. Even for the $\mathrm{H}_{2} \mathrm{O}$ dimer it was hard to converge them; we had to use very large angular basis sets with $j_{A}^{\max }=j_{B}^{\max }=13$. The results 




Fig. 4 VRT levels of the $\mathrm{D}_{2} \mathrm{O}$ dimer corresponding to the intermolecular vibrations, calculated from the CC-pol-8s potential, in comparison with experimental data. ${ }^{27,38}$ All symbols are explained in Fig. 3, except for $\mathrm{DT}^{2}$ which denotes the donor torsion overtone $\left(A^{\prime}\right){ }^{39}$ In ref. 28 , this mode was referred to as in-plane bend (IB).

Table 10 Root mean square relative percentage errors of various properties of $\mathrm{H}_{2} \mathrm{O}$ and $\mathrm{D}_{2} \mathrm{O}$ dimers from calculations with different potentials, compared with experimental data from ref. 29 and 30 and references therein. The following properties are used in this analysis: ground state rotational constants $A$ and $B+C$, ground state tunneling splittings $a(0)+a(1)$ (acceptor switch), $i_{1}$ and $i_{2}$ (donor acceptor interchange), and frequencies of the intermolecular vibrations DT, $\mathrm{AW}, \mathrm{AT}$, and $\mathrm{DT}^{2}$ for $K=0$

\begin{tabular}{|c|c|c|c|c|}
\hline Potential & Rotational constants $^{a}$ & $a(0)+a(1)$ & $i_{1}, i_{2}$ & Vibrational frequencies \\
\hline \multicolumn{5}{|l|}{$\mathrm{H}_{2} \mathrm{O}$ dimer } \\
\hline TTM $2.1^{b}$ & $16.0 / 17.0$ & 82 & 67 & 12 \\
\hline $\mathrm{VRT}$ ASP-W)III ${ }^{c}$ & $6.6 / 5.1$ & 0.1 & 55 & 6.9 \\
\hline $\mathrm{HBB}^{d}$ & $0.05 / 1.4$ & 19 & 1.3 & 3.6 \\
\hline CC-pol ${ }^{e}$ & $2.2 / 3.1$ & 19 & 5.9 & 4.3 \\
\hline CC-pol-8s ${ }^{f}$ & $1.9 / 1.7$ & 9.8 & 2.5 & 2.1 \\
\hline CC-pol-8s ${ }^{g}$ & $1.0 / 0.6$ & 10.1 & 3.2 & 2.3 \\
\hline \multicolumn{5}{|l|}{$\mathrm{D}_{2} \mathrm{O}$ dimer } \\
\hline VRT(ASP-W)III $^{c}$ & 2.2 & 11 & 64 & 4.5 \\
\hline $\mathrm{HBB}^{d}$ & 1.7 & 31 & 1.2 & 4.5 \\
\hline CC-pol ${ }^{e}$ & 3.6 & 34 & 14 & 4.1 \\
\hline CC-pol-8s ${ }^{f}$ & 3.3 & 12 & 5.2 & 1.3 \\
\hline CC-pol-8s ${ }^{g}$ & 0.6 & 12 & 7.3 & 1.7 \\
\hline
\end{tabular}

${ }^{a}$ Relative to the experimental values of $A$ for the $\mathrm{H}_{2} \mathrm{O}$ dimer of $7.44 / 7.59 \mathrm{~cm}^{-1}$, respectively. ${ }^{b}$ Potential from ref. $40 .{ }^{c}$ Potential from ref. 16. ${ }^{d}$ From ref. 10. ${ }^{e}$ From ref. 3. ${ }^{f}$ VRT levels computed with the method of Groenenboom et al., neglecting off-diagonal Coriolis coupling. ${ }^{g}$ VRT levels computed with the method of Leforestier et al., including off-diagonal Coriolis coupling. 
Table 11 Bifurcation shifts (in $\left.\mathrm{cm}^{-1}\right)$ for $\left(\mathrm{H}_{2} \mathrm{O}\right)_{2}$ in the ground state calculated with $j_{A}^{\max }=j_{B}^{\max }=13$ for various potentials. The parameters are defined in eqns (10) and (11)

\begin{tabular}{|c|c|c|c|c|c|c|}
\hline Potential & $b_{1}(K=0)$ & $b_{2}(K=0)$ & $b_{1}(|K|=1)$ & $b_{2}(|K|=1)$ & $b_{1}^{01}$ & $b_{2}^{01}$ \\
\hline $\begin{array}{l}\mathrm{HBB}^{a} \\
\text { CC-pol }\end{array}$ & $\begin{array}{l}0.0460 \\
0.0157\end{array}$ & $\begin{array}{l}0.0460 \\
0.0137\end{array}$ & & & & \\
\hline $\begin{array}{l}\text { CC-pol-8s } \\
\text { Experimental }^{c}\end{array}$ & 0.0218 & 0.0216 & -0.0198 & -0.0255 & $\begin{array}{l}0.0208 \\
0.0227\end{array}$ & $\begin{array}{l}0.0236 \\
0.0249\end{array}$ \\
\hline
\end{tabular}

are given and compared with experiment in Table 11. The values measured ${ }^{29}$ are the differences of the shifts of the levels with $|K|=1$ and those of the levels with $K=0$ and it was assumed that the shifts for $|K|=1$ are nearly the opposite of the shifts for $K=0$, so that $b_{1}^{(01)} \approx b_{1}^{(0)}$ and $b_{2}^{(01)} \approx b_{2}^{(0)}$. We tested this assumption by calculating the shifts both for $K=0$ and for $|K|=1$; our results show that it is approximately correct. The difference shifts computed can be compared directly to the measured values. We find good agreement for CC-pol-8s, much better than for the CC-pol and HBB potentials, see Table 11.

\section{Conclusions}

The performance of various site-site functional forms in fitting a set of $a b$ initio interaction energies for the water dimer has been investigated. The exponential plus $1 / r^{n}$ form of eqns (1)-(3) has been found to represent a good compromise between the accuracy of fits and smooth behavior between grid points. The much more flexible B-spline form can decrease the RMSE by about a factor of two if very large numbers of splines are used, but at this level of accuracy introduces spurious undulations between grid points so that the predictions in such regions are inaccurate.

It has been found that the most effective way to increase the accuracy of this type of fits is to use a large number of sites with carefully optimized positions. The B-spline basis was found very useful in such optimizations since each optimization step was reduced to a linear least-square problem. The final potential has eight symmetry-distinct sites per monomer and is denoted as CC-pol-8s. Once the site positions were established, the site-site potentials of the form of eqns (1)-(3) were fitted using a nonlinear least-square procedure.

The CC-pol-8s potential has been shown to perform better than any published potential to date in recovering the barriers related to the characteristic points on the surface (with the average deviation decreased by about a factor of five). Also in predicting spectra of both the $\mathrm{H}_{2} \mathrm{O}$ and $\mathrm{D}_{2} \mathrm{O}$ dimer, it performs significantly and systematically better (with deviations from experiment on average decreased by a factor of two to three compared to previously best predictions, and more dramatic improvements for properties of the vibrationally excited states). The high accuracy of CC-pol-8s allowed us to predict, for the first time, the bifurcation shifts in the spectra, reaching excellent agreement with experiment. In fact, the agreement between theory and experiment for the complete spectrum is unprecedented for a system of such size, so we may say that CC-pol-8s becomes the new benchmark water dimer potential.
The higher accuracy of CC-pol-8s compared to CC-pol required the use of a more complicated functional form. Since the time of calculations for a site-site fit is proportional to the square of the number of sites, one would expect an order of magnitude increase in costs. However, since the additional sites are of a simpler form (contain only the exponential terms) and due to a more optimal coding, the ratio of times is only a factor of about three. The Fortran source code of the CC-pol8 s potential is contained in the ESI $\dagger$ associated with this work. We have also included there routines for evaluating three-body nonadditive interaction energies from ref. 44 and $N$-body induction (polarization) contributions, thus allowing calculations of the interaction potential in any water cluster or in condensed phases of water.

The major neglected effect in the description of the water dimer by the CC-pol-8s potential is due to monomer flexibility. We are working on inclusion of this effect via an adaptation of the flexible-monomer water dimer potential from ref. 42.

This work was supported by the NSF grant CHE-0555979 and by the Nederlandse Organisatie voor Wetenschappelijk Onderzoek (NWO).

\section{References}

1. R. Bukowski, K. Szalewicz, G. C. Groenenboom and A. van der Avoird, J. Chem. Phys., 2008, 128, 094313.

2. R. Bukowski, K. Szalewicz, G. C. Groenenboom and A. van der Avoird, Science, 2007, 315, 1249.

3. R. Bukowski, K. Szalewicz, G. C. Groenenboom and A. van der Avoird, J. Chem. Phys., 2008, 128, 094314.

4. B. Jeziorski, R. Moszynski and K. Szalewicz, Chem. Rev., 1994, 94, 1887.

5. E. M. Mas, K. Szalewicz, R. Bukowski and B. Jeziorski, J. Chem. Phys., 1997, 107, 4207.

6. E. M. Mas, R. Bukowski, K. Szalewicz, G. C. Groenenboom, P. E. S. Wormer and A. van der Avoird, J. Chem. Phys., 2000, 113, 6687.

7. G. C. Groenenboom, P. E. S. Wormer, A. van der Avoird, E. M. Mas, R. Bukowski and K. Szalewicz, J. Chem. Phys., 2000, 113, 6702 .

8. G. C. Groenenboom, E. M. Mas, R. Bukowski, K. Szalewicz, P. E. S. Wormer and A. van der Avoird, Phys. Rev. Lett., 2000, 84, 4072.

9. R. Bukowski, K. Szalewicz, G. C. Groenenboom and A. van der Avoird, J. Chem. Phys., 2006, 125, 044301.

10. X. Huang, B. J. Braams, J. M. Bowman, R. E. A. Kelly, J. Tennyson, G. C. Groenenboom and A. van der Avoird, J. Chem. Phys., 2008, 128, 034312.

11. X. Huang, B. J. Braams and J. M. Bowman, J. Phys. Chem. A, $2006,110,445$.

12. W. R. Johnson, S. A. Blundell and J. Sapirstein, Phys. Rev. A: At., Mol., Opt. Phys., 1998, 37, 307.

13. G. S. Tschumper, M. L. Leininger, B. C. Hoffman, E. F. Valeev, H. F. Schaefer III and M. Quack, J. Chem. Phys., 2002, 116, 690

14. W. Klopper, J. G. C. M. van Duijneveldt-van de Rijdt and F. B. van Duijneveldt, Phys. Chem. Chem. Phys., 2000, 2, 2227. 
15. R. S. Fellers, C. Leforestier, L. B. Braly, M. G. Brown and R. J. Saykally, Science, 1999, 284, 945.

16. N. Goldman, R. S. Fellers, M. G. Brown, L. B. Braly, C. J. Keoshian, C. Leforestier and R. J. Saykally, J. Chem. Phys., 2002, 116, 10148.

17. J. A. Odutola and T. R. Dyke, J. Chem. Phys., 1980, 72, 5062.

18. E. M. Mas and K. Szalewicz, J. Chem. Phys., 1996, 104, 7606.

19. C. Leforestier, L. B. Braly, K. Liu, M. J. Elrod and R. J. Saykally, J. Chem. Phys., 1997, 106, 8527.

20. R. S. Fellers, L. B. Braly, R. J. Saykally and C. Leforestier, J. Chem. Phys., 1997, 110, 6306.

21. H. Chen, S. Liu and J. C. Light, J. Chem. Phys., 1999, 110, 168.

22. E. Zwart, J. J. ter Meulen and W. L. Meerts, J. Mol. Spectrosc., $1990,173,115$

23. E. Zwart, J. J. ter Meulen, W. L. Meerts and L. H. Coudert, J. Mol. Spectrosc., 1991, 147, 27.

24. G. T. Fraser, Int. Rev. Phys. Chem., 1991, 10, 189

25. E. N. Karyakin, G. T. Fraser and R. D. Suenram, Mol. Phys., 1993, 78, 1179

26. J. B. Paul, R. A. Provencal and R. J. Saykally, J. Phys. Chem. A, 1998, 102, 3279.

27. L. B. Braly, J. D. Cruzan, K. Liu, R. S. Fellers and R. J. Saykally, J. Chem. Phys., 2000, 112, 10293.

28. L. B. Braly, K. Liu, M. G. Brown, F. N. Keutsch, R. S. Fellers and R. J. Saykally, J. Chem. Phys., 2000, 112, 10314.

29. F. N. Keutsch, N. Goldman, H. A. Harker, C. Leforestier and R. J. Saykally, Mol. Phys., 2003, 101, 3477.

30. F. N. Keutsch, L. B. Braly, M. G. Brown, H. A. Harker, P. B. Petersen, C. Leforestier and R. J. Saykally, J. Chem. Phys., 2003, 119, 8927.
31. C. Leforestier, F. Gatti, R. Fellers and R. Saykally, J. Chem. Phys., 2002, 117, 8710.

32. D. M. Brink and G. R. Satchler, Angular Momentum, Clarendon, Oxford, 3rd edn, 1993.

33. H. C. Longuet-Higgins, Mol. Phys., 1963, 6, 445.

34. D. T. Colbert and W. H. Miller, J. Chem. Phys., 1992, 96, 1982.

35. G. C. Groenenboom and D. T. Colbert, J. Chem. Phys., 1993, 99, 9681.

36. D. O. Harris, G. G. Engerholm and W. D. Gwinn, J. Chem. Phys., $1965, \mathbf{4 3}, 1515$

37. C. Leforestier, J. Chem. Phys., 1994, 101, 7357.

38. L. B. Braly, Ph.D. thesis, University of California at Berkeley, 1999.

39. M. J. Smit, G. C. Groenenboom, P. E. S. Wormer, A. van der Avoird, R. Bukowski and K. Szalewicz, J. Phys. Chem. A, 2001, 105,6212 .

40. G. S. Fanourgakis and S. S. Xantheas, J. Phys. Chem. A, 2006, 110, 4100

41. J. M. Bowman, C. Leforestier, R. van Harrevelt and A. van der Avoird, 2008, to be published.

42. K. Szalewicz, G. Murdachaew, R. Bukowski, O. Akin-Ojo and C. Leforestier, in Lecture Series on Computer and Computational Science: ICCMSE 2006, ed. G Maroulis and T Simos, Brill Academic Publishers, Leiden, 2006, vol. 6, pp. 482-491.

43. M. Jeziorska, P. Jankowski, K. Szalewicz and B. Jeziorski, J. Chem. Phys., 2000, 113, 2957.

44. E. M. Mas, R. Bukowski and K. Szalewicz, J. Chem. Phys., 2003, 118, 4386. 